\title{
RESEARCH
}

Open Access

\section{Wnt-driven LARGE2 mediates laminin- adhesive O-glycosylation in human colonic epithelial cells and colorectal cancer}

Vanessa Dietinger ${ }^{1,2,3 \dagger}$, Cira R. García de Durango ${ }^{1,2,3+}$, Svenja Wiechmann ${ }^{1,2,4}$, Sophie L. Boos ${ }^{1,2,3}$, Marlies Michl', Jens Neumann ${ }^{5}$, Heiko Hermeking ${ }^{1,2,5}$, Bernhard Kuster ${ }^{1,2,4,6}$ and Peter Jung ${ }^{1,2,3,7^{*}}$ (i)

\begin{abstract}
Background: Wnt signaling drives epithelial self-renewal and disease progression in human colonic epithelium and colorectal cancer (CRC). Characterization of Wnt effector pathways is key for our understanding of these processes and for developing therapeutic strategies that aim to preserve tissue homeostasis. O-glycosylated cell surface proteins, such as a-dystroglycan (a-DG), mediate cellular adhesion to extracellular matrix components. We revealed a Wnt/LARGE2/a-DG signaling pathway which triggers this mode of colonic epithelial cell-to-matrix interaction in health and disease.

Methods: Next generation sequencing upon shRNA-mediated silencing of adenomatous polyposis coli (APC), and quantitative chromatin immunoprecipitation (qChIP) combined with CRISPR/Cas9-mediated transcription factor binding site targeting characterized LARGE2 as a Wnt target gene. Quantitative mass spectrometry analysis on sizefractionated, glycoprotein-enriched samples revealed functional O-glycosylation of a-DG by LARGE2 in CRC. The biology of Wnt/LARGE2/a-DG signaling was assessed by affinity-based glycoprotein enrichment, laminin overlay, CRC-to-endothelial cell adhesion, and transwell migration assays. Experiments on primary tissue, human colonic (tumor) organoids, and bioinformatic analysis of CRC cohort data confirmed the biological relevance of our findings. (Continued on next page)
\end{abstract}

\footnotetext{
* Correspondence: p.jung@dkfz-heidelberg.de

${ }^{\dagger}$ Vanessa Dietinger and Cira R. García de Durango contributed equally to this work.

${ }^{1}$ German Cancer Research Center (DKFZ), Heidelberg, Germany

${ }^{2}$ German Cancer Consortium (DKTK), Partner site Munich, Munich, Germany

Full list of author information is available at the end of the article
}

(c) The Author(s). 2020 Open Access This article is licensed under a Creative Commons Attribution 4.0 International License, which permits use, sharing, adaptation, distribution and reproduction in any medium or format, as long as you give appropriate credit to the original author(s) and the source, provide a link to the Creative Commons licence, and indicate if changes were made. The images or other third party material in this article are included in the article's Creative Commons licence, unless indicated otherwise in a credit line to the material. If material is not included in the article's Creative Commons licence and your intended use is not permitted by statutory regulation or exceeds the permitted use, you will need to obtain permission directly from the copyright holder. To view a copy of this licence, visit http://creativecommons.org/licenses/by/4.0/ The Creative Commons Public Domain Dedication waiver (http://creativecommons.org/publicdomain/zero/1.0/) applies to the data made available in this article, unless otherwise stated in a credit line to the data. 
(Continued from previous page)

Results: Next generation sequencing identified the LARGE2 O-glycosyltransferase encoding gene as differentially expressed upon Wnt activation in CRC. Silencing of APC, conditional expression of oncogenic $\beta$-catenin and endogenous $\beta$-catenin-sequestration affected LARGE2 expression. The first intron of LARGE2 contained a CTTGATC motif essential for Wnt-driven LARGE2 expression, showed occupation by the Wnt transcription factor TCF7L2, and Wnt activation triggered LARGE2-dependent a-DG O-glycosylation and laminin-adhesion in CRC cells. Colonic crypts and organoids expressed LARGE2 mainly in stem cell-enriched subpopulations. In human adenoma organoids, activity of the LARGE2/a-DG axis was Wnt-dose dependent. LARGE2 expression was elevated in CRC and correlated with the Wnt-driven molecular subtype and intestinal stem cell features. O-glycosylated a-DG represented a Wnt/ LARGE2-dependent feature in CRC cell lines and patient-derived tumor organoids. Modulation of LARGE2/a-DG signaling affected CRC cell migration through laminin-coated membranes and adhesion to endothelial cells.

Conclusions: We conclude that the LARGE2 O-glycosyltransferase-encoding gene represents a direct target of canonical Wnt signaling and mediates functional O-glycosylation of a-dystroglycan (a-DG) in human colonic stem/ progenitor cells and Wnt-driven CRC. Our work implies that aberrant Wnt activation augments CRC cell-matrix adhesion by increasing LARGE/a-DG-mediated laminin-adhesiveness.

Keywords: O-glycosylation, LARGE2, Wnt signaling, Colorectal cancer, Organoid, Colonic stem cell

\section{Background}

Wnt signaling plays a pivotal role in human colonic stem cell (hCoSC) and colorectal cancer (CRC) biology $[1,2]$, and constitutive Wnt activation in CRC frequently arises from mutations in the tumor suppressor gene $A P C$. Wnt activity in APC mutant CRC cells at least partially depends on the length of truncated APC [3]. The majority of tumors harbor $A P C$ alleles altered in the mutation cluster region (MCR), and the encoded variants of truncated APC retain one or several 20 amino acid repeat (20*AAR) $\beta$-catenin binding sites, thus preventing full Wnt activation [4-6]. During CRC progression, Wnt signaling is frequently augmented by crosstalk with other corrupted signaling pathways $[7,8]$ or by extrinsic cues from the tumor microenvironment (TME) [9]. A concise characterization of effectors driven by activated Wnt signaling in a dose-dependent manner at different disease stages will help our understanding of CRC progression.

Upon Wnt activation, nuclear translocation of $\beta$ catenin and its association with TCF/LEF transcription factors leads to transcriptional regulation of target genes [10]. In the intestinal tract and in CRC, the $\beta$-catenin/ TCF7L2 complex and its downstream target genes mediate epithelial tissue self-renewal $[1,11]$. Importantly, $\beta$ catenin was initially found to control cell adhesion at adherens junctions [12]. APC itself interacts with cytoskeletal components, and its genetic alteration affects cell adhesion and migration [13]. Besides this, Wnt signaling drives expression of extracellular matrix (ECM) proteins, such as fibronectin [14] and laminin [15]. In return, differential interactions of intestinal stem cells (ISCs) and CRC cells with the extracellular matrix (ECM) contribute to acquisition of epithelial stemness and metastatic tumor traits, respectively [16].
The outer membrane protein $\alpha$-dystroglycan $(\alpha-D G)$ and the transmembrane protein $\beta$-dystroglycan $(\beta-D G)$ are proteolytic cleavage products of the same propeptide DAG1, and O-glycosylated $\alpha$-DG functions as a receptor for laminin-domain containing ECM protein ligands, such as laminin, agrin, and neurexin [17, 18]. At least 23 gene products are involved in the process of $\mathrm{O}$ mannosyl-glycan synthesis on $\alpha-D G$, and their loss of function contributes to the pathology of congenital muscular dystrophies [18]. Once a core glycosyl structure on $\alpha$-DG has been generated by priming enzymes, the glycosyltransferases LARGE1 or its paralog LARGE2 (a.k.a. GYLTL1B) polymerize a glucosaminoglycan disaccharide on phosphorylated O-linked mannose, often referred to as matriglycan, which is essential for $\alpha-D G$ binding to laminin [18-20]. While LARGE1 is essential for $\alpha-D G$ O-glycosylation in heart, brain, and skeletal muscle, a functional necessity for LARGE2 regarding this process exists in kidney and prostate [21, 22]. However, the biology of LARGE2 in the human colon and in CRC is largely unknown.

Here, we report a direct link between canonical Wnt signaling and the LARGE2-dependent, O-glycosylationmediated laminin adhesion in human colonic epithelium and in CRC. By using the human colon (tumor) organoid model $[2,23]$ and CRISPR/Cas9-mediated genome editing, we addressed the consequences of physiologic extrinsic and oncogenic intrinsic Wnt activation for this process.

\section{Methods}

\section{Cell culture}

Cell lines KM12c and KM12-L4a were a kind gift from Professor I. Fidler (MD Anderson, TX, USA). HT-29, 
SW620, LS174T, Colo205, KM12c/L4a, and HEK293T (ATCC, LGC Standards, Wesel, Germany) were maintained in Dulbecco's Modified Eagle Medium supplemented with 10\% FBS (both Gibco, Thermo Fisher Scientific, MA, USA) and $1 \%$ penicillin/streptomycin (Gibco). SW480 cells were cultivated in RPMI-1640 medium (Gibco), supplemented with 10\% FBS and 1\% penicillin/streptomycin (Gibco). RKO cells were maintained in McCoy's 5A (Sigma, Merck, Darmstadt, Germany) supplemented with $10 \% \mathrm{FBS}$ and $1 \%$ penicillin/streptomycin (Gibco). HMEC-I cells were kindly provided by PD Dr. Thomas Grünewald (DKFZ Heidelberg) and maintained in RPMI-1640 medium supplemented with $50 \mathrm{ng} / \mathrm{ml}$ human epidermal growth factor (EGF, PeproTech, NJ, USA) and $1 \mu \mathrm{g} / \mathrm{mL}$ hydrocortison (Sigma, Merck). Cultures were kept at $37^{\circ} \mathrm{C}, 5 \% \mathrm{CO}_{2}$ and subcultured as needed. All cell lines were regularly tested negative for Mycoplasma contamination by using the LookOut Mycoplasma PCR detection kit (Sigma, Merck).

\section{Next generation sequencing (RNA-Seq) analysis and bioinformatic data processing}

For RNA-Seq, total RNA was isolated from HT-29 cells using the PureLink RNA purification kit and PureLink DNase (both Invitrogen, Thermo Fisher) according to the manufacturer's protocol. Quality of isolated RNA was confirmed with the Agilent BioAnalyzer 2100 (Agilent, CA, USA). Sequencing libraries were prepared using the TrueSeq Stranded mRNA Library Prep Kit for Illumina (New England Biolabs, MA, USA) according to the manufacturer's instructions. $50 \mathrm{bp}$ single-read sequencing was performed on a HiSeq 2000 v4 (Illumina, CA, USA) according to the manufacturer's protocol. For the bioinformatic data analyses, base calling was done with bcl2fastq 2.19.0.316. For all samples, low quality bases were removed with Fastq_quality_filter from the FASTX Toolkit 0.0.13 (http://hannonlab.cshl.edu/fastx_ toolkit/index.html) with $90 \%$ of the read needing a quality phred score $>20$. Homertools 4.7 [24] were used for PolyA-tail trimming, and reads with a length $<17$ were removed. Genomic mapping was performed with TOPHAT2 [25] for filtered reads with human genome assembly 38 and PicardTools 1.78 CollectRNASeqMetrics (https://broadinstitute.githu.io/picard/). Count data were generated by htseq-count [26] using the gencode.v29.annotation.gtf (https://www.gencodegenes.org/) file for annotation. For the comparison with DESeq2 [27], the input tables containing the replicates for the groups to compare were created by a custom perl script. In the count matrix, rows with an average count number $<10$ were removed. Then, DESeq2 (version 1.4.1) was run with default parameters. The results tables were annotated with gene information (gene symbol, gene type) derived from the gencode.v29.annotation.gtf file.

\section{Gene expression data and gene set enrichment analysis (GSEA)}

Gene expression data of COAD, READ and PRAD were obtained from GDC-TCGA datasets [28], and another set covering 566 CRC cases was derived from Marisa et al. [29] (GSE35982). Data of 515 transplanted human tumor samples (PDX) from 244 patients were included from the Isella et al. (GSE76402) data set [30]. Information to assign the samples with the CMS subtypes are described in Guinney et al. [31] and the CRIS categories are described in Isella et al. [30]

LARGE2 gene signatures were generated from GDCTCGA datasets as well as from the PDX microarray data described by Isella et al. GSEA was performed as described in Subramanian et al. [32] using the GSEApreRanked tool of the GSEA 3.0 Desktop Application (Broad Institute, http://www.gsea-msigdb.org/gsea/index. jsp).

\section{Chromatin Immunoprecipitation and analysis}

SW480 cells were used for ChIP analysis according to manufacturer's protocol (SimpleChIP ${ }^{\circ}$ Enzymatic Chromatin IP Kit \#9003, Cell signaling Technology). $10 \mu \mathrm{g}$ of fragmented DNA were incubated over night with either TCF7L2 (1:50, C48H11) or control rabbit IgG (1:500, \#2729) antibody (both Cell Signaling Technology). The precipitated chromatin fragments were purified, and analyzed via $\mathrm{qRT}-\mathrm{PCR}$ using primer listed in Additional file 13.

\section{CRISPR/Cas9-mediated engineering of APC mutant human} adenoma organoids

Isolation of crypts from patient material and 3D cultivation as patient-derived organoids (PDOs) was performed as previously described [2], and colonic PDOs were transfected by lipofection as has been reported [33]. In brief, PDOs grown in WREN medium (Wnt3a, RSPO-3 and Noggin conditioned media derived from L-WNR cells, CRL-3276, ATCC) and Advanced DMEM/F12 (ADF, Gibco) 50:50, supplemented with Glutamax, 10 mM HEPES, N-2 [1×], B-27 without retinoic acid [1 $\times$ ] (Invitrogen, Thermo Fisher), $1 \mathrm{mMN}$-acetylcysteine (Sigma, Merck), $50 \mathrm{ng} / \mathrm{ml}$ recombinant human epidermal growth factor (PeproTech), $7.5 \mu \mathrm{M}$ SB202190 (Sigma, Merck) and $10 \mu \mathrm{M}$ Y27632 (SelleckChem, TX, USA) were disaggregated with TripLE Select (Gibco, Thermo Fisher) for $10 \mathrm{~min}$ at $37^{\circ} \mathrm{C}$. Single PDO cells were resuspended in WREN medium without antibiotics, supplemented with Rho kinase inhibitor Y-27632 $(10 \mu \mathrm{M})$ and plated in 24-well low attachment plates $(160,000$ cells per well) already containing $200 \mu \mathrm{l}$ of lipocomplexes. 
Ribonucleoparticles consisting of Cas9 and single guide (sg) RNA were prepared according to manufacturer specifications (IDT, IA, USA), and the cell/enzyme mixture was centrifuged for one hour at $32^{\circ} \mathrm{C}$, followed by 4h of incubation at $37^{\circ} \mathrm{C} / 5 \% \mathrm{CO}_{2}$. Cells were then plated in Matrigel (BD Corning, NY, USA) and kept in WREN medium for three days prior to selection. Selection for ADOs was achieved by omitting Wnt and RSPO from the culture medium (EN medium).

\section{Patient-derived tumor organoid (PDTO) isolation and culture}

Tissue pieces from primary CRC or liver metastasis were cut into small pieces and one fraction was processed for subsequent immunohistochemistry analysis. The remaining tissue was incubated with Normocin (Invivogen) and Antibiotic-Antimycotic (Thermo Fisher) for 15 min at room temperature. Next, pieces were resuspended in disaggregation media (ADF (Thermo Fisher), $5 \mathrm{U} / \mathrm{ml}$ Dispase (Stem Cell Technologies, Vancouver, Canada), 75 $\mathrm{U} / \mathrm{ml}$ Collagenase IV (Gibco, Thermo Fisher) and $10 \mu \mathrm{M}$ Y-27632 (SelleckChem) and incubated at $37^{\circ} \mathrm{C}$ for $30 \mathrm{~min}$ before passing through a $1.2 \mathrm{~mm}$ needle. Once small cell clusters were obtained, the suspension was passed through $70 \mu \mathrm{m}$ cell strainer (BD, NJ, USA) and freed from erythrocytes using ammonium-choride buffer. CRC cells were resuspended in Matrigel, plated in 24-well tissue culture plates and overlaid with tumor organoid culture (TOC) medium (ADF supplemented with $10 \mathrm{mM}$ HEPES, Glutamax, 1x B27 (all Thermo Fisher), $1 \mathrm{mMN}$-acetylcysteine (Sigma, Merck), $50 \mathrm{ng} / \mathrm{mL}$ recombinant human epidermal growth factor (EGF, PeproTech), $0.015 \mu \mathrm{M}$ Prostaglandine E2 (PGE2, Sigma, Merck), $25 \mathrm{ng} / \mathrm{mL}$ human Noggin (PeproTech), $7.5 \mu \mathrm{M}$ SB202190 and $0.5 \mu \mathrm{M}$ LY2157299 (Selleckchem), and $50 \mu \mathrm{g} / \mathrm{mL}$ Normocin (Invitrogen). $10 \mu \mathrm{M}$ Y27632 (SelleckChem) was added for $48 \mathrm{~h}$ to avoid anoikis. Medium was replaced every $2-3$ days. For serial passaging, PDTOs were disaggregated using 0.025\% Trypsin (Gibco, Thermo Fisher) and subsequently passed through a $0.8 \mathrm{~mm}$ needle. After washing with ADF medium, PDTO cells and small cell aggregates were resuspended in Matrigel and solidified drops were overlaid with TOC medium.

\section{Immuno-labeling of human CRC cells and PDOs for flow cytometry-assisted cell sorting (FACS)}

For surface abundance analysis of O-glycosylated $\alpha$-DG, cells were detached with $8 \mathrm{mM}$ EDTA/PBS, washed and resuspended in staining buffer (PBS/5\% FBS). Cells were stained with IIh6c4 antibody (1:100, Millipore/Merck) for one hour on ice, followed by labelling with a FITCcoupled secondary antibody (1:200, Jackson ImmunoResearch, UK, Cat.No: 515-095-062). Control cells were stained with the secondary antibody alone. DAPI
$(0.3 \mu \mathrm{g} / \mathrm{ml}$, Carl Roth, Karlsruhe, Germany) was added to exclude dead cells. Analysis was performed on a LSR Fortessa (BD) instrument, and data analysis was done with FlowJo software (BD).

Labeling and sorting of PDO cells was performed as described previously [34]. In brief, human colonic organoids were disaggregated using Dispase II solution (Sigma, Merck), and cells resuspended in FACS buffer (ADF, $10 \mathrm{mM}$ HEPES, $10 \mathrm{mM}$ Glutamax, 5\% FBS, $10 \mu \mathrm{M}$ Y-27632). Cells were stained with Allophycocyanin (APC)-coupled anti-PTK7 antibodies (1:20, Cat.No: CCK-4, Miltenyi, Bergisch Gladbach, Germany,). Viable, DAPI-negative cell fractions were used to determine auto-fluorescence and to specify the "negative", nonstaining cell population for FACS gating. The brightest 14-15\% PTK7+ cells were sorted as the PTK7-high fraction. The PTK7-low population comprised the 25-30\% PTK7+ cells adjacent to the PTK7-high population, while the PTK7-negative subpopulation did not stain for PTK7. Cell sorting was performed on a FACS Aria III instrument (BD) and analysis was performed with FlowJo software. After cell sorting, the different fractions were pelleted by centrifugation and re-suspended in $500 \mu \mathrm{l}$ Trizol (Invitrogen, Thermo Fisher) for RNA isolation and gene expression analyses via $\mathrm{TaqMan}^{\mathrm{Tm}}$ gene expression assays (Applied Biosystems, Thermo Fisher, see Additional File 13 for probe IDs).

Sub-fractionation of human colonic crypt cells was performed essentially as described previously [2] by immuno-labelling of epithelial crypt single cells with anti-EPHB2 antibody (1:100, BD Biosciences, clone $2 \mathrm{H} 9$, APC-coupled). Viable control cells (DAPI-negative fraction) were used to define the EPHB2-negative cell population.

\section{WGA-AE purification, immunoblot analysis of glycoproteins and laminin overlay assay}

Whole cell protein lysates were prepared from subconfluent cultures using WGA Lysis Buffer $(50 \mathrm{mM}$ Tris/HCl, pH 7.4 (Sigma), $150 \mathrm{mM} \mathrm{NaCl}$ (Carl Roth), 1\% Triton X-100 (AppliChem, Darmstadt, Germany) and Protease Inhibitors (Sigma, Merck), and protein concentration was determined with Bradford Reagent (Sigma, Merck). For precipitation of glycoproteins, $1-3 \mathrm{mg}$ of lysate was incubated with $50 \mu \mathrm{l}$ equilibrated WGA agarose beads (Vector labs, CA, USA) overnight at $4{ }^{\circ} \mathrm{C}$ while rotating. Glycoproteins were eluted from WGA beads by heating for $10 \mathrm{~min}$ at $70^{\circ} \mathrm{C}$ in $60 \mu \mathrm{l}$ of $2 \mathrm{x}$ Laemmli buffer. Whole cell or glycoprotein-enriched lysates were separated on $8-10 \%$ SDS-acrylamide gels and proteins transferred to Immobilon PVDF membranes (Millipore, Merck). For the laminin overlay assay, membranes were blocked in 5\% skim milk in laminin binding buffer (LBB; $19 \mathrm{mM}$ triethanolamine, $140 \mathrm{mM} \mathrm{NaCl}, 1 \mathrm{mM} \mathrm{MgCl}_{2}, 1$ 
$\mathrm{mM} \mathrm{CaCl} 2, \mathrm{pH}$ 7.4) for one hour at room temperature. After washing in 3\% BSA/LBB, membranes were incubated with $5 \mu \mathrm{g} / \mathrm{mL}$ Matrigel (contains $60 \%$ laminin-111) in $3 \% \mathrm{BSA} / \mathrm{LBB}$ at $4{ }^{\circ} \mathrm{C}$ overnight. Unbound residual Matrigel/laminin-111 was washed of the membrane carefully with $5 \%$ milk in LBB. For immunodetection, membranes were incubated with the following antibodies: anti-APC (1:500, Ali 12-28, SantaCruz Biotech), anti-actin (1:2000, A2066, Sigma, Merck), anti-tubulin (1:2000, T9026, Sigma, Merck), anti- $\alpha$ DG (1:500, IIh6c4, Millipore, Merck), anti-DAG1 (1:500, 11,017-1-AP, PTGLAB, Manchester, UK), anti-laminin (1:2000, NB300-144, Novus Bio, MN, USA). Chemiluminescence signals from horseradish-peroxidase (HRP) coupled secondary antibodies (1:10000, Jackson ImmunoResearch) were generated using Immobilon Western HRP Substrate (MerckMillipore) and detected with a Laser Scanning System (Odyssey Fc, LI-COR, NE, USA).

\section{LC-MS/MS analysis of WGA-IP enriched glycoproteins}

Glycoprotein enriched lysates were prepared as described above. Proteins bound to WGA agarose beads were eluted with LDS sample buffer (NuPAGE, Thermo Fisher) containing $100 \mathrm{mM}$ DTT for $10 \mathrm{~min}$ at $70^{\circ} \mathrm{C}$ and alkylated with $55 \mathrm{mM}$ CAA for $30 \mathrm{~min}$ at RT. WGA eluates were purified and fractionated into six fractions by NuPAGE Bis-Tris Gels (Thermo Fisher) and subsequently subjected to in-gel tryptic digestion according to standard procedures. After drying in a centrifugal evaporator, the samples were stored at $-80^{\circ} \mathrm{C}$ until LC-MS/MS analysis.

Nano-flow LC-MS/MS measurement of peptides in eluates was performed using a nanoLC UltiMate 3000 (Thermo Fisher) coupled to a quadrupole-Orbitrap Q Exactive HF mass spectrometer (Thermo Fisher). Peptides were desalted on a trap column $(100 \mu \mathrm{m} \times 2 \mathrm{~cm}$, packed in-house with Reprosil-Pur C18-AQ $5 \mu \mathrm{m}$ resin; Dr. Maisch $\mathrm{GmbH}$, Ammerbuch-Entringen, Germany) in $0.1 \% \mathrm{FA}$ at $5 \mu \mathrm{l} / \mathrm{min}$ and separated on an analytical column $(75 \mu \mathrm{m} \times 40 \mathrm{~cm}$, packed in-house with ReprosilPur C18-AQ, $3 \mu \mathrm{m}$ resin; Dr. Maisch) using a $22 \mathrm{~min}$ linear gradient from 4 to $32 \%$ solvent B $(0.1 \%$ formic acid, $5 \%$ DMSO in acetonitrile) in solvent A (0. $1 \%$ formic acid, $5 \%$ DMSO in water) at a flow rate of $300 \mathrm{~nL} / \mathrm{min}$. The mass spectrometer was operated in data dependent acquisition and positive ionization mode. MS1 spectra were acquired over a range of $360-1300 \mathrm{~m} / \mathrm{z}$ at a resolution of 60,000 in the Orbitrap by applying an AGC of $3 \mathrm{e} 6$ or maximum injection time of $10 \mathrm{~ms}$. Up to 10 peptide precursors were selected for fragmentation by higher energy collision-induced dissociation (HCD; 1.7 $\mathrm{m} / \mathrm{z}$ isolation window, AGC value of $1 \mathrm{e} 5$, maximum injection time of $25 \mathrm{~ms}$ ) using $25 \%$ normalized collision energy (NCE) and analyzed at a resolution of 15,000 in the Orbitrap (Thermo Fisher).

\section{Transwell migration assay}

To analyze invasion, cell inserts $(0.8 \mu \mathrm{m}$ pore size), were coated with $1 \mu \mathrm{g} / \mathrm{mL}$ Laminin 111 from EngelbrethHolm-Swarm (EHS) lathrytic mouse tumor (Santa Cruz Biotechnology, TX, USA) for $1 \mathrm{~h}$ at room temperature. $1 \times 10^{5}$ cells, deprived of serum for at least $24 \mathrm{~h}$, were seeded in serum free DMEM on laminin in the upper chamber. As chemo-attractant, DMEM, supplemented with $10 \%$ FBS, was placed in the bottom chamber. After 24-48 h, cells on the upper side of the membrane were removed using a cotton swab. After fixation in 4\% PFA for $20 \mathrm{~min}$, the membrane was air dried and stained with $100 \mathrm{ng} / \mathrm{mL}$ DAPI in $0.1 \%$ Triton X-100. Images were captured with a Zeiss LSM 700 confocal microscope in five random fields per membrane and migrated cells counted with ImageJ. Experiments were performed three times in duplicates. Relative migration was normalized to the corresponding control.

\section{CRC cell adhesion assay on HMEC-1 cells}

$2 \times 10^{5}$ HMEC- 1 cells per 48-well were seeded to form a confluent endothelial monolayer within $48-72 \mathrm{~h}$. Separately cultured, sub-confluent KM12-L4a-Luc CRC cells (stably transduced with pV2-Luc2, kindly provided by Prof. Andreas Trump, DKFZ Heidelberg, and wt or KO for LARGE2) were detached carefully with Accutase, adjusted to $0,75 * 10^{6}$ single cells $/ \mathrm{mL}$, and $200 \mu \mathrm{l}$ of this suspension was added to confluent HMEC-I cells for the indicated time. Next, supernatants were removed and short-term co-cultures were washed twice with PBS. Cells were lysed in $50 \mu \mathrm{l} 1 \mathrm{x}$ Passive Lysis Buffer (Promega, WI, USA), lysates were spun down and $10 \mu \mathrm{l}$ was used for measurement on a Berthold Orion II Microplate Luminometer after adding $50 \mu \mathrm{l}$ Beetle Juice (pjk Biotech, Kleinblittersdorf, Germany). Three experiments were performed independently on different days (biological triplicates) and each sample was measured three times (technical triplicates).

\section{Immunohistochemistry}

Immunohistochemical (IHC) staining was carried out on $2 \mu \mathrm{m}$ formalin-fixed, paraffin-embedded sections using standard procedures. In brief, antigen retrieval was achieved with target retrieval solution (S1699, Agilent Technologies) via microwave heating. Incubation with the primary antibody IIh6 (Santa Cruz) at a concentration of $4 \mu \mathrm{g} / \mathrm{ml}$ was done at room temperature for one hour. As a detection system, biotinylated anti-mouse IgM (BA2020, Vector Labs) and streptavidin-HRP (RE 7104, Novocastra, Newcastle, UK) was used. Samples were developed via exposure to 3,3'-diaminobenzidine (DAB+, K3468, Agilent) and counterstained with hematoxylin Gill's Formula (H-3401, Vector Labs). For IHC staining against $\alpha$-DG on mouse FFPE tissue, 
Crystal MausBlock (Fa. DCS, Hamburg, Germany, ML125R015) was used to avoid non-specific binding of the secondary antibody. Processed slides were scanned on a Vectra Polaris ${ }^{\mathrm{Tx}}$ slide scanner using 40-fold scan resolution and snapshots taken via Phenochart 1.0.8 software (both AKOYA Biosciences, MA, USA).

\section{In situ hybridization}

Detection of Large 2 mRNA in mouse ileum normal mucosa and ApcMin-driven adenoma was performed as described before [35]. In brief, RNA probes were created with DIG RNA Labeling Kit (Roche) according to the manufacturers' protocol. $5 \mu \mathrm{m}$ thick FFPE sections of mouse tissues were de-waxed and re-hydrated using standard procedures. Proteins were digested by treating the slides with $0.2 \mathrm{~N} \mathrm{HCl}$, followed by incubation with $30 \mu \mathrm{g} / \mathrm{mL}$ Proteinase $\mathrm{K}$ in PBS at $37^{\circ} \mathrm{C}$. Digestion was stopped with $0.2 \%$ Glycine/PBS. Sections were post-fixed in 4\% PFA for $10 \mathrm{~min}$, washed in PBS three times and histones acetylated with acetic anhydride solution $(1.5 \%$ triethanolamine, $0.15 \% \mathrm{HCl}, 0.6 \%$ acetic anhydride). Slides were washed and incubated for one hour at $65{ }^{\circ} \mathrm{C}$ in pre-hybridization solution, containing 50\% Formamide, 5X SSC pH 4.5, 2\% Blocking Reagent (Roche), 0.05\% CHAPS, $5 \mathrm{mM}$ EDTA, $50 \mu \mathrm{g} / \mathrm{ml}$ Heparin and $50 \mu \mathrm{g} / \mathrm{ml}$ yeast RNA (all Sigma, Merck). Probes were used in a concentration of $500 \mathrm{ng}$ per labeling, diluted in prehybridization solution and incubated for $24 \mathrm{~h}$ at $65^{\circ} \mathrm{C}$. Post-hybridization washes were performed using 50\% Formamide in 2x SSC Buffer pH 4.5, three times á 20 min at $65^{\circ} \mathrm{C}$. Sections were then rinsed in Tris/ $\mathrm{NaCl}$ buffer and blocked for $30 \mathrm{~min}$ in blocking solution $(0.5 \%$ blocking powder in Tris/NaCl). Sheep anti-DIG antibody (Roche, Basel, Switzerland) was diluted 1:2000 in blocking solution and incubated overnight at $4{ }^{\circ} \mathrm{C}$. Finally, samples were first washed in Tris/ $\mathrm{NaCl}$ buffer and then in NTM buffer $(0.1 \mathrm{M}$ Tris $\mathrm{pH} 9.5,0.1 \mathrm{M} \mathrm{NaCl}, 0.05 \mathrm{M}$ $\mathrm{MgCl}_{2}$ ) before developing the sections in BCIP/NBT Liquid Substrate (Roche) for $48 \mathrm{~h}$. Processed slides were scanned on a Vectra Polaris ${ }^{\mathrm{TM}}$ slide scanner using 40-fold scan resolution and snapshots taken via Phenochart 1.0.8 software (both AKOYA Biosciences).

\section{Statistical analysis}

GraphPad Prism software (v7.01) was used for statistical analyses. For calculation of significant differences between two groups of biological replicates, a Student's t-test (unpaired, two-tailed, 5\% FDR) was applied. For qRT-PCR data, standard deviation (SD, $n \geq 3)$ was calculated as indicated. For the comparison of 4-5 patient cohort subpopulations (CMS and CRIS classification), a multiple comparison one-way ANOVA test was applied.
For calculation of correlation coefficients, Pearson's correlation analysis was applied.

\section{Results}

Silencing of truncated APC activates Wnt target gene expression in CRC cells

The 1555 amino acid variant of APC in HT-29 and Colo205 CRC cells encompasses three 20 AARs (3*20AAR) sufficient for partial $\beta$-catenin inactivation, which translates to low cell-intrinsic Wnt activity $[4,36]$. Stable transduction with lentiviruses encoding for a doxycycline (DOX)-inducible short hairpin RNA (shRNA) allowed us to silence $A P C$ mRNA and protein in these cell lines (Fig. 1A,B and Additional file 2A-C). $A P C$ silencing augmented expression of bona-fide Wnt target genes, such as AXIN2 [37] and LGR5 [38] (Additional file 2A,C). Next generation sequencing (NGS, RNA-Seq) $72 \mathrm{~h}$ after shRNA-mediated $A P C$ silencing in HT-29 cells revealed 205 upregulated genes, while the expression of 82 genes was downregulated ( $>4$ fold change, $p$-value $<0.01$ ) (Fig. $1 \mathrm{C}$ and Additional file 3). Besides the known Wnt targets AXIN2, NOTUM, SP5, $N K D 1$, and $A S C L 2$, we found that additional candidate genes, such as PTK7 and LARGE2, were upregulated in this setting (Fig. 1C and Additional file 3).

LARGE2 represents a direct target of Wnt signaling in CRC Since a direct link between canonical Wnt signaling and O-glycosylation has not been reported yet to our knowledge, we focused our work on $L A R G E 2$, a gene encoding for a bifunctional O-glycosyltransferase [22]. Induction of $L A R G E 2$ and bona-fide Wnt target genes upon $A P C$ silencing in HT-29 and Colo205 cells was confirmed by quantitative real-time PCR (qRT-PCR) (Fig. 1D and Additional file 2C, D). Expression of $L A R G E 1$, the paralog of LARGE2, was low (Ct values of 32-33 versus $\sim 28$ for $L A R G E 2)$ in these cells, and $L A R G E 1$ was not affected by $A P C$ silencing (Fig. 1D). Since APC possesses functionalities beyond controlling Wnt activity [39], we stably transduced HT-29 cells with lentiviruses carrying a DOX-inducible allele of oncogenic $\beta$-catenin (CTNNB1-S33Y) [40]. CTNNB1-S33Y triggered expression of $L A R G E 2$, suggesting that $L A R G E 2$ is driven by canonical Wnt signaling (Fig. 1E). To interfere with $\beta$-catenin/TCF functionality in Wnt-active CRC cell lines, we stably transduced LS174T and SW480 to express the CTNNB1-binding domain of TCF7L2 (nTCF: amino acids 1 to 90 ) fused to a tamoxifen-inducible version of the hormone-binding domain of the estrogen receptor (ERT2) (here referred to as nTCF-ERT2 or, as a cell line suffix, $-\mathrm{NE}$ ). Cells stably expressing the ERT2 domain (referred to as -ERT) alone served as a control. Upon addition of 4-hydroxy-tamoxifen (4-OHT) to cells 


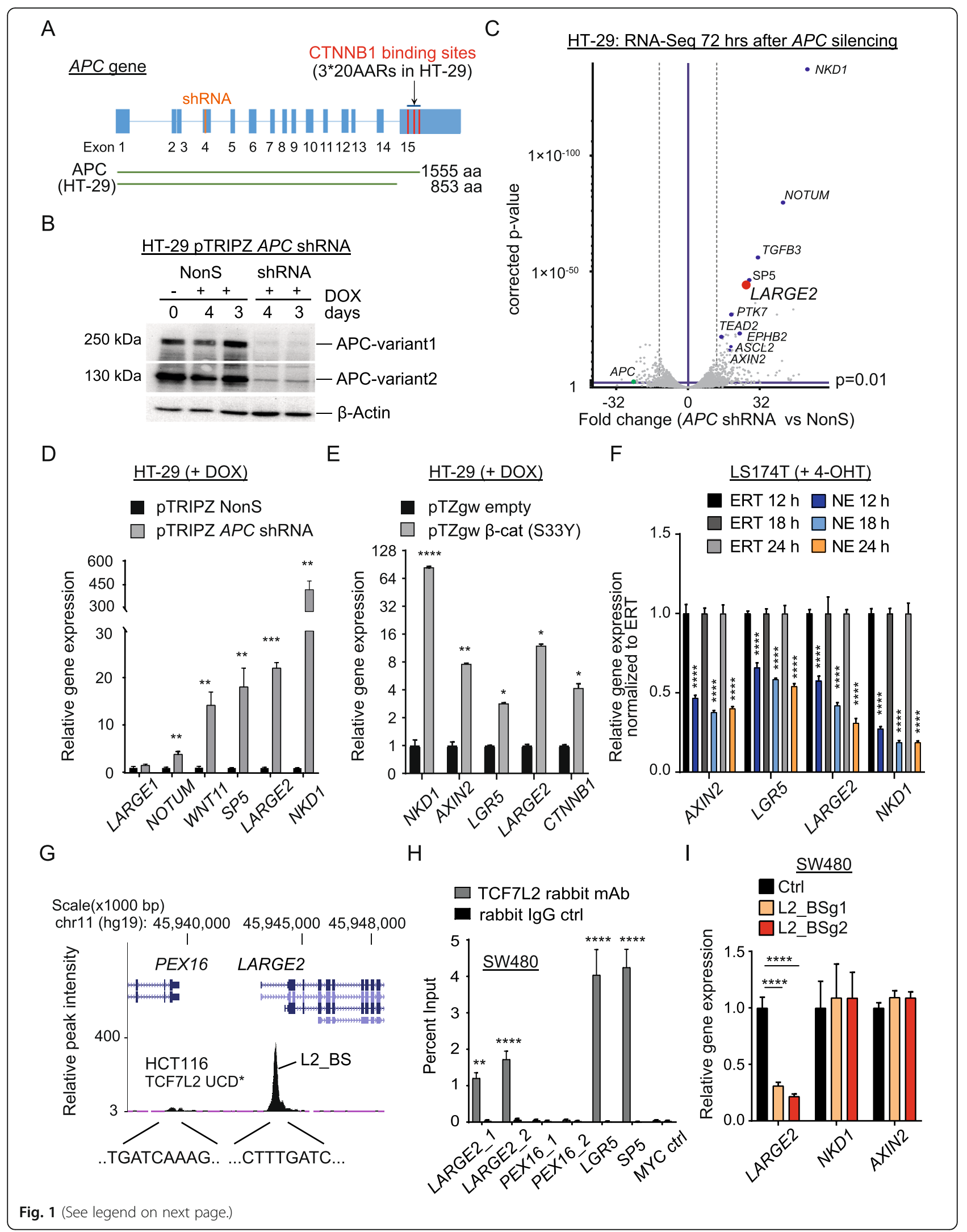


(See figure on previous page.)

Fig. 1 LARGE2 is a direct target of Wnt signaling in colorectal cancer. A) APC gene structure, exons indicated as blue bars. A short hairpin RNA (shRNA, indicated in orange) was used to silence APC. Truncated APC variants in HT-29 CRC cells are shown as green lines. aa: amino acids. B) Immunoblot analysis of APC and $\beta$-actin on WCL from HT-29 cells treated with $500 \mathrm{ng} / \mathrm{ml}$ DOX to induce expression of APC-targeting shRNA or a non-silencing shRNA (NonS). C) Volcano plot showing genes deregulated $72 \mathrm{~h}$ after silencing of APC in HT-29 cells, as analyzed via RNA-Seq on biological duplicates. Cut off: normalized $p$-value $<0.01$ (horizontal blue line), fold change $>4$ (vertical dotted lines). D,E) qRT-PCR analysis of indicated genes up-regulated upon conditional APC silencing (D) or expression of S33Y-mutated CTNNB1 (E) in HT-29 cells for $72 \mathrm{~h}$ (+ $500 \mathrm{ng} / \mathrm{ml}$ DOX). Results are shown as mean $\pm \mathrm{SD}(n=3) ;{ }^{*} p<0.05$; ${ }^{* *} p<0.01$; ${ }^{* * *} p<0.0001$. F) qRT-PCR analysis of indicated genes in LS174T-NE or $-\mathrm{E}$ cells after treatment with $400 \mathrm{nM} 4-\mathrm{OHT}$ for the indicated times. Results are shown as mean $\pm \mathrm{SD}(\mathrm{n}=3) ;{ }^{* * *} \mathrm{p}<0.0001$. G) Structure of the proximal LARGE2 genomic locus (UCSC Browser) showing a potential TCF7L2 binding site (L2_BS) in the first LARGE2 intron. * Source: HCT-116 TCF7L2 UC Davis ChIP-seq Signal from ENCODE/SYDH (Peggy Farnham lab). H) qChIP analysis on genomic DNA from SW480 cells. The amount of DNA immunoprecipitated with TCF7L2 antibody or rabbit lgG-control in each sample is shown as percentage of chromatin input. Results are shown as mean \pm SD $(n=3) ;{ }^{* *} p<0.01 ;{ }^{* * *} p<0.0001$. I) qRT-PCR analysis of indicated genes in stably transduced SW480 cells upon CRISPR/ eCas9-mediated targeting of the TCF7L2_BS in the first intron of LARGE2 via two different guide RNAs. Results are shown as mean \pm SD $(n=5)$; **** $p<0.0001$

expressing nTCF-ERT2, which then sequesters nuclear $\beta$-catenin to nTCF7 unable to bind DNA, LARGE2 expression was downregulated to a similar extent as LGR5 and AXIN2 when compared to ERT2 control cells (Fig. $1 \mathrm{~F}$, Additional file 2E,F).

In silico analysis of putative TCF7L2 genomic DNA binding loci in HCT116 CRC cells via the UCSC genome browser revealed DNA occupation by TCF7L2 within the first intron of LARGE2 (ENCODE annotation data [41], Farnham-USC, Accession No: ENCSR000EUV) (Fig. 1G). Quantitative chromatin immunoprecipitation (qChIP) analysis in SW480 cells confirmed binding of TCF7L2 to the first intron of $L A R G E 2$, which contains a canonical TCF7L2 binding motif CTTTGATC [42] (Fig. $1 \mathrm{G}, \mathrm{H})$. A second potential binding motif $\sim 5 \mathrm{~kb}$ upstream of LARGE2 did not show occupation by these factors (Fig. 1G,H). To demonstrate the specificity of our qChIP assay, we confirmed TCF7L2 within the LGR5 and SP5 promoters, while an amplicon upstream of $c$ MYC previously shown to lack TCF7L2 and $\beta$-catenin occupancy [43] was not enriched (Fig. 1H). We next assessed the functionality of the TCF7L2 binding site within LARGE2 (L2_BS) by performing CRISPR/Cas9mediated mutagenesis: Stable delivery of a Streptococcus pyogenes Cas9 derivative engineered for improved specificity (referred to as eCas9) [44] plus two different guideRNAs (BSg1 and BSg2) recognizing a sequence either on the $(+)$ or the $(-)$ strand of the L2_BS was achieved by lentiviral transduction. As a control, a non-targeting tracrRNA was used. Successful targeting of the L2_BS in stably transduced SW480 cell pools was confirmed by a mutation detection assay (Additional file 2G). Indeed, CRISPR/eCas9-mediated L2_BS targeting led to 4- to 5fold reduced LARGE2 mRNA levels and abolished L2 BS occupation by TCF7L2 in SW480 (Fig. 1I and Additional file $2 \mathrm{H})$. Accordingly, site directed mutagenesis of the "CTTTGATC" TCF7L2 binding motif to CTTTGGCC [45] within a $\sim 600$ bp ectopic DNA fragment of LARGE2 compromised the activity of a luciferase reporter (Additional file 2I). Overall, our data suggest that Wnt signaling triggers LARGE2 gene expression via the $\beta$-catenin/TCF7L2 transcriptional complex in CRC.

\section{LARGE2 correlates with active Wnt signaling and $\mathrm{hCoSC}$ gene expression in CRC}

Considering a well-accepted segregation of colon cancer into four distinct sub-groups known as the consensus molecular subtypes (CMS) [31], we wondered if expression of LARGE2 was associated with one of these categories. When analyzing two independent, CMS classified colon cancer patient cohorts (TCGA-COAD [46] and Marisa et al. (2013) [29]), we found highest LARGE2 mRNA levels in CMS2, specified by an active WNT/ MYC program [31] (Fig. 2A,B). Since contaminating stroma cells within tissue samples were shown to bias this transcriptional classification of CRC [47, 48], Isella and colleagues defined five purely epithelial CRC Intrinsic Subtypes (CRIS A-E) from gene expression profiles of 515 xeno-transplanted human tumor samples (PDXs), which they had derived from 244 individuals suffering from CRC [30]. By analyzing these data, we found $L A R G E 2$ gene expression was explicitly enriched in CRIS-D tumors characterized by an intestinal stem cell (ISC) phenotype and high Wnt activity [30] (Fig. 2C). Moreover, Isella and colleagues used their algorithm to re-stratify the TCGA-COAD [46] and the Marisa et al.(2013) [29] cohorts into the five CRIS classes [30]. Also here, LARGE2 was enriched in the highly Wnt-active and ISC-like CRIS subtype D (Additional file 4A,B).

Next, we used NGS RNA-Seq data derived from 458 colon cancer and 167 rectal tumors from TCGA as provided by the NCI-GDC [28], and we generated preranked lists of genes according to their Pearson correlation with $L A R G E 2$ gene expression (data not shown). By GSEA, we found that two independent sets of Wnt target genes $[15,49]$ and a recently described oncogenic/ intrinsic Wnt signature [50] were enriched among the 
A

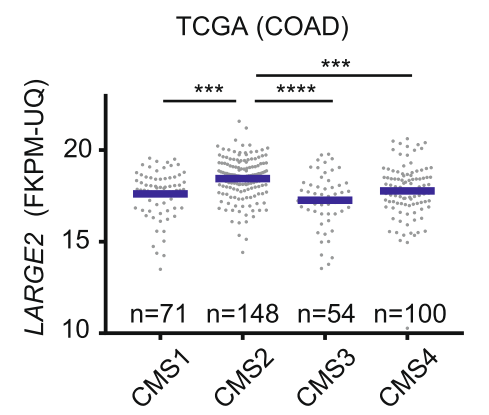

D

Consensus Molecular Subtype

Wnt-up gene signature

(61 genes; Herbst et al., 2014)

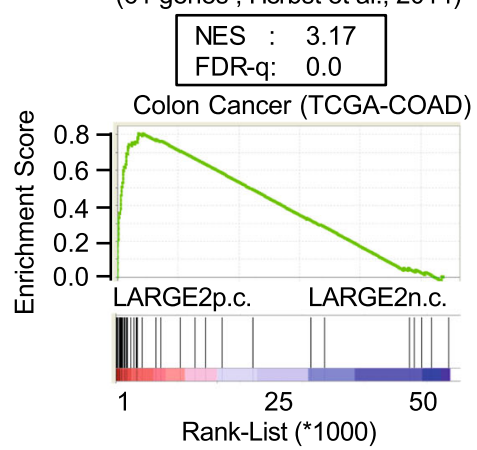

G

PTK7+ hCoSC signature

(327 genes ; Jung et al., 2015)

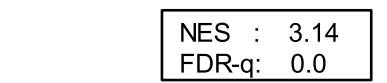

Colon Cancer (TCGA-COAD).

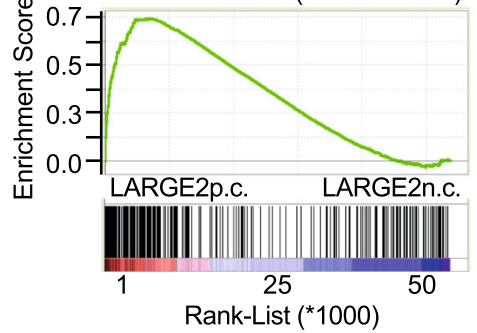

J

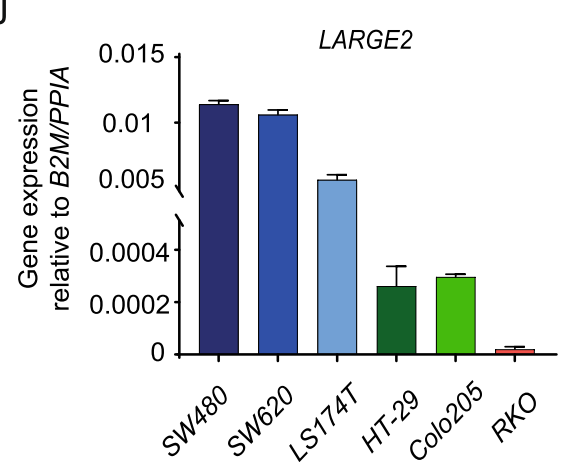

E

$\mathrm{H}$
B

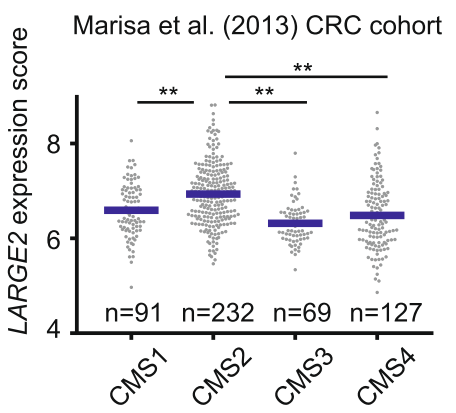

Consensus Molecular Subtype

Wnt-up gene signature

(61 genes ; Herbst et al., 2014)

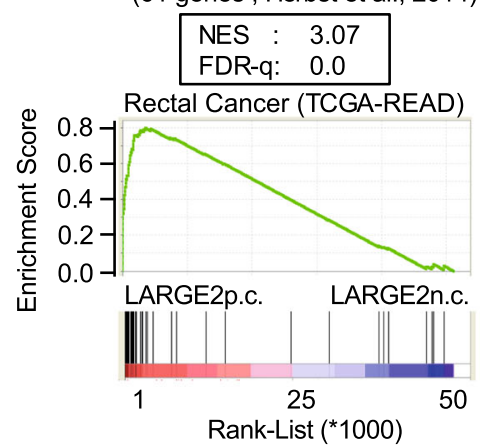

Wnt-up gene signature

(61 genes ; Herbst et al., 2014)

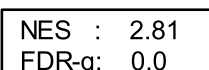

CRC (PDXs, Isella et al.)
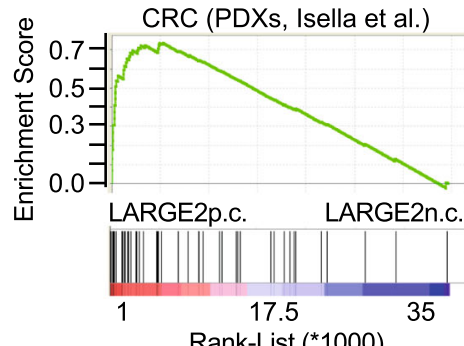

K

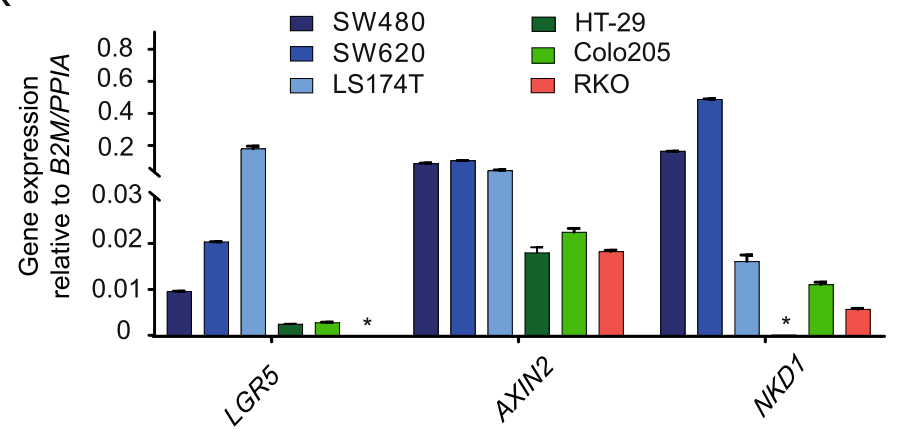

C

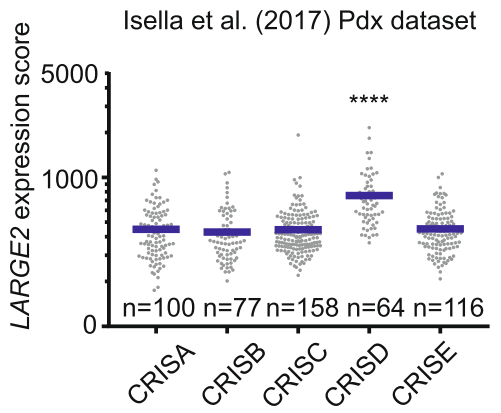

CRC intrinsic subtype

(59 genes ; Jung et al., 2011)

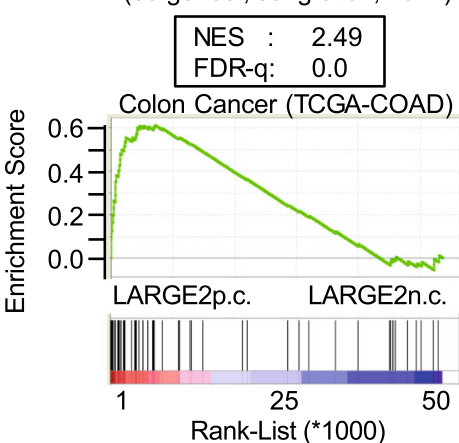

I

Wnt-up gene signature

(61 genes ; Herbst et al., 2014)

\begin{tabular}{ll|}
\hline NES : & 2.37 \\
FDR-q: & 0.0
\end{tabular}

$58 \mathrm{CRC}$ cell lines (CCLE)
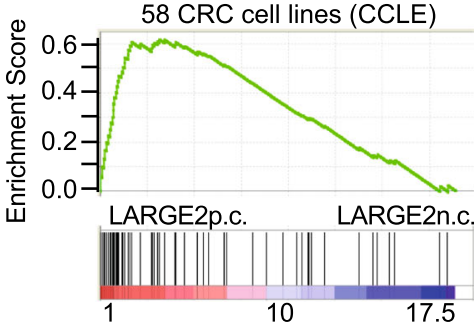

Rank-List $\left({ }^{* 1000)}\right.$
$\mathrm{EPHB} 2+\mathrm{hCoSC}$ signature

Fig. 2 (See legend on next page.) 
(See figure on previous page.)

Fig. 2 LARGE2 mRNA levels in CRC correlate with high Wnt activity and hCoSC gene expression. (A-C) LARGE2 gene expression in consensus molecular subtypes (CMS) of the TCGA-COAD cohort $(n=373)(\mathbf{A})$, the Marisa et al. (2013) CRC cohort $(n=519)$ (B), and in the CRC intrinsic subtypes (CRIS) of a PDX cohort published by Isella et al. (2017) $(n=515)$ (C). Shown are the mean levels of LARGE2 (blue horizontal line). Asterisks indicate a significant difference between CMS2 or CRISD and the other subtypes. Significance was calculated by one-way ANOVA testing (** $p<$ $\left.0.01,{ }^{* * *} p<0.001,{ }^{* * *} p<0.0001\right)$. D-G) GSEA on LARGE2 gene signatures derived from TCGA-COAD and TCGA-READ RNA-Seq data sets. Shown are enrichments of Wht target gene sets upregulated upon siRNA-mediated $\beta$-catenin silencing in CRC cells (D, E), and gene sets which specify $\mathrm{EPHB}_{2}{ }^{\text {high }}(\mathbf{F})$ or PTK $7^{\text {high }}(\mathbf{G})$ hCoSCs (see method section for details). NES: normalized enrichment score, FDR-q: False discovery rate q-value. $\left.\mathbf{H}, \mathbf{l}\right)$ GSEA on LARGE2 gene signatures derived from microarray data of a PDX CRC cohort published by Isella et al. 2017 (H) and the 58 CRC lines included in the CCLE database (I). p.c.: positive correlation, n.c.: negative correlation. J,K) qRT-PCR analysis of LARGE2 gene expression (J) or expression of bona-fide Wnt target genes (K) in the indicated CRC cell lines. Analysis was performed in duplicates (two independent RNA samples per cell line). Error bars indicate \pm SD. * gene expression not detectable by qRT-PCR

genes positively correlated with LARGE2 expression (Fig. 2D,E and Additional file 4C-E). Since Wnt signaling represents a major driver of stemness in colonic epithelium and CRC [51], we studied the enrichment of two published gene sets specifying EPHB2 ${ }^{\text {high }}$ and $\mathrm{PTK} 7^{\text {high }}$ hCoSCs [2, 34] in the LARGE2 signature of CRC: Both $\mathrm{hCoSC}$-specific gene sets were enriched among the genes positively correlated with LARGE2 expression (Fig. 2F,G and Additional file 4F,G). We obtained similar results with a pre-ranked list of $L A R G E 2$ correlated genes derived from the CRIS gene expression data set $(n=515$ PDX CRC samples) [30] (Fig. $2 \mathrm{H}$ and Additional file 4H$\mathrm{J})$ and from microarray data $(n=58$ established CRC cell lines) obtained from the Cancer Cell Line Encyclopedia (CCLE) [52] (Fig. 2I and Additional file $4 \mathrm{~K}, \mathrm{~L}$ ). Accordingly, HT-29 and Colo205, specified by low intrinsic Wnt activity [36], and RKO cells, wild-type for $A P C$ and CTNNB1 [53], displayed lower levels of $L A R G E 2, L G R 5$, $A X I N 2$, and NKD1 expression when compared to highly Wnt-active SW480 and SW620 cells which harbor shorter APC variants [4], or LS174T which express oncogenic mutant $\beta$-catenin [54] (Fig. 2J,K). These data show that LARGE2 is enriched in colorectal tumors characterized by active Wnt signaling, an ISC phenotype, and a hCoSC gene expression program.

\section{LARGE2 is essential and sufficient for matriglycan formation on a-DG in CRC}

To address the relevance of LARGE2 for functional $\alpha$ DG O-glycosylation in CRC, we generated targeted knock-outs (KOs) of LARGE2 in CRC cell lines and a patient-derived tumor organoid (PDTO) line PDTO1 (Additional file 5) by CRISPR/eCas9-mediated genome editing using two guide RNAs directed against different regions of the $L A R G E 2$ open reading frame (Fig. 3A, upper panel). Due to the lack of commercially available antibodies against LARGE2, we confirmed successful gene editing by performing a mismatch detection assay (Fig. 3A, lower panel). Next, we purified glycoproteinenriched fractions from $L A R G E 2$ wild-type and $L A R G E 2$ KO whole cell lysates (WCLs) via wheat germ agglutinin (WGA) agarose-affinity enrichment (-AE). Immunoblot analysis with an O-glycosylation sensitive antibody against $\alpha$-DG (IIh6c4) revealed that targeting LARGE2 indeed diminished O-glycosylated $\alpha$-DG at 150-250 kDa in SW480, SW620 (Fig. 3B, left panel), PDTO1 (Fig. $3 \mathrm{~B}$, right panel), and LS174T (Fig. 3C). Transient delivery of Cas9 plus a sgRNA against LARGE2 in SW620 cells yielded the same result (Additional file 6A). Importantly, LARGE2 targeting abolished laminin binding capacity of WGA-AE purified glycoprotein fractions in the laminin overlay assay, suggesting that LARGE2 is essential to create functional matriglycan on $\alpha-D G$ in CRC (Fig. 3C). Loss of LARGE2 did not affect DAG1 mRNA or $\beta$-dystroglycan ( $\beta$-DG) protein (Fig. 3B,C, Additional file $6 \mathrm{~A}, \mathrm{~B})$.

To answer if LARGE2 alone was sufficient for matriglycan formation on $\alpha-D G$ in CRC cells characterized by low Wnt activity and hence low endogenous LARGE2 levels, we stably equipped HT-29 cells with a DOXinducible $L A R G E 2$ cDNA by lentiviral transduction. In this model, addition of DOX for $72 \mathrm{~h}$ led to $\sim 10-25$ fold higher levels of LARGE2 mRNA when compared to Wnt-active SW480 and PDTO1 cells or Wnt-activated HT-29 cells (Additional file 6C). Since ectopic LARGE2 has been reported to also $\mathrm{O}$-glycosylate laminin-binding and IIh6c4-reactive glypican 4 (GPC4) in mouse ES cells [55], we performed quantitative mass spectrometry (LCMS/MS) analysis on WGA-AE purified glycoproteins from these cells. Due to the shift in molecular weight (MW) of proteins upon O-glycosylation by LARGE2, we fractionated electrophoretically separated samples into 6 MW windows prior to LC-MS/MS analysis (Additional file 6D). LARGE2 was detected in the 75-110 $\mathrm{kDa}$ window, in accordance with its calculated MW of $81.8 \mathrm{kDa}$, and DAG1 peptides from control HT-29 cells occurred at $0-55 \mathrm{kDa}$ and $75-110 \mathrm{kDa}$ (Fig. 3D). Upon ectopic expression of LARGE2, peptides matching the $\alpha$ DG domain of DAG1, but not $\beta$-DG, appeared at 110$160 \mathrm{kDa}$ and with higher intensity at $160-300 \mathrm{kDa}$ (Fig. $3 \mathrm{D}$, Additional file 7). This $160-300 \mathrm{kDa}$ fraction also contained unique peptides for LAMA2 (Laminin subunit alpha 2), but LAMA2 was absent in control samples (Fig. 3D). This shows that HT-29 cells express LAMA2, 
A

LARGE2 genomic locus

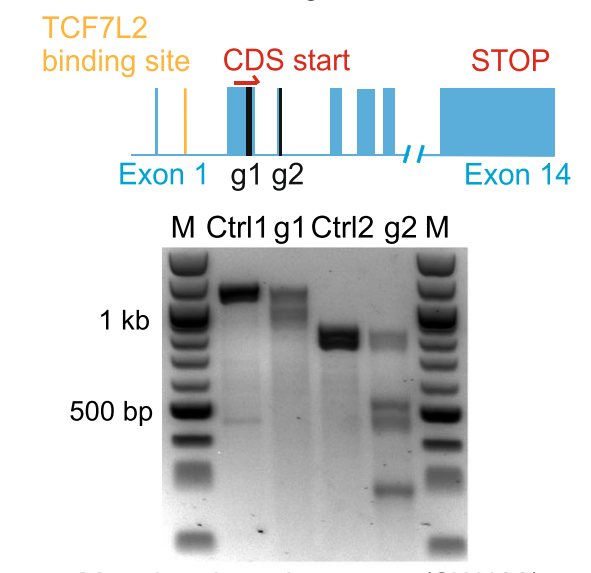

C

Mutation detection assay (SW480)

\section{$\underline{\text { LS174T }}$}

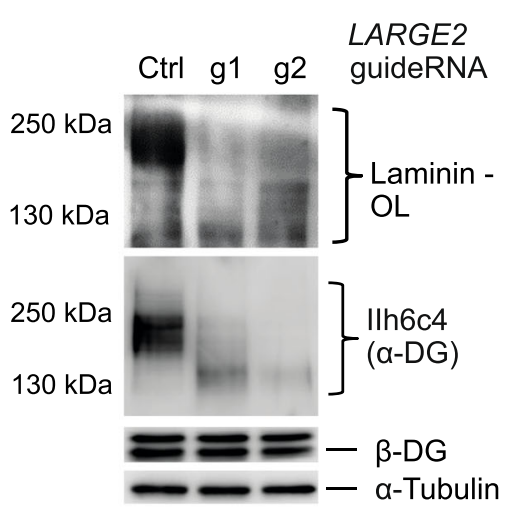

$E$

HT-29 pTZgw-LARGE2

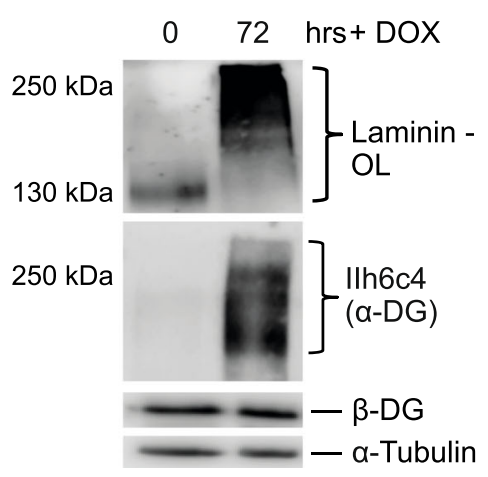

B

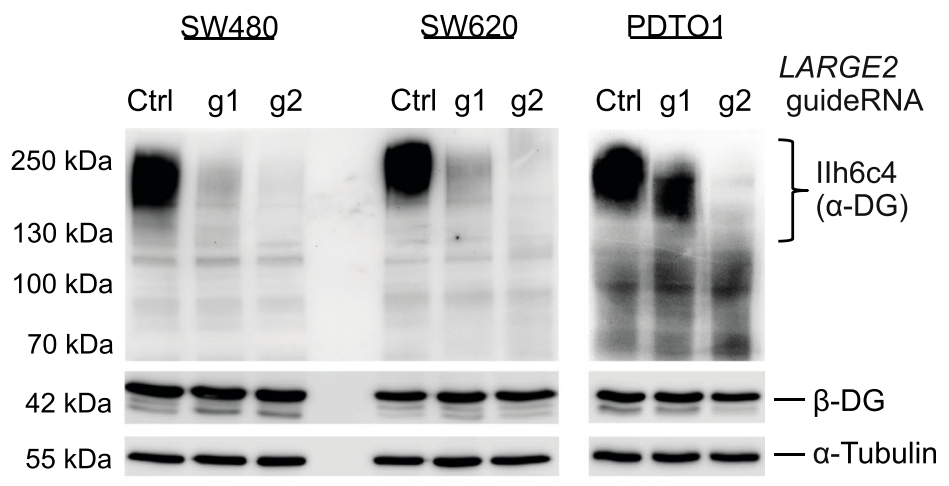

D

\begin{tabular}{|c|c|c|}
\hline $\begin{array}{c}\text { HT-29 } \\
\text { WGA-IP fraction } \\
\text { for LC-MS/MS }\end{array}$ & $\begin{array}{c}\frac{\text { pTZgw-empty }}{\text { Protein ID }} \\
\text { (iBAQ) }\end{array}$ & $\begin{array}{l}\text { pTZgw-LARGE2 } \\
\text { Protein ID (iBAQ) }\end{array}$ \\
\hline B1 $(160<k D$ & & $\begin{array}{l}\text { DAG1* }(54.0 \mathrm{E}+06) \\
\text { LAMA2 }(0.45 \mathrm{E}+06)\end{array}$ \\
\hline $\mathrm{B} 2(110-160 \mathrm{kD})$ & & DAG1* $(11.3 \mathrm{E}+06)$ \\
\hline B3 (75 - 110 kD) & $\begin{array}{l}\text { DAG1 }(83.4 \mathrm{E}+06) \\
\text { LARGE2 }(3.5 \mathrm{E}+07)\end{array}$ & $\begin{array}{l}\text { DAG1 }(11.9 \mathrm{E}+06) \\
\text { LARGE2 }{ }^{* *}(1.5 \mathrm{E}+11)\end{array}$ \\
\hline B4 (55 - 75 kD) & & \\
\hline B5 (25 - $55 \mathrm{kD})$ & DAG1 $(15.9 E+06)$ & DAG1 $(25.0 \mathrm{E}+06)$ \\
\hline $\mathrm{B} 6(<25 \mathrm{kD})$ & DAG1 (37.2E+06) & DAG1 (29.5E+06) \\
\hline
\end{tabular}

*: all detected DAG1 peptides matched alpha-DG

**: intensity maximum of ectopic LARGE2

$\mathrm{F}$

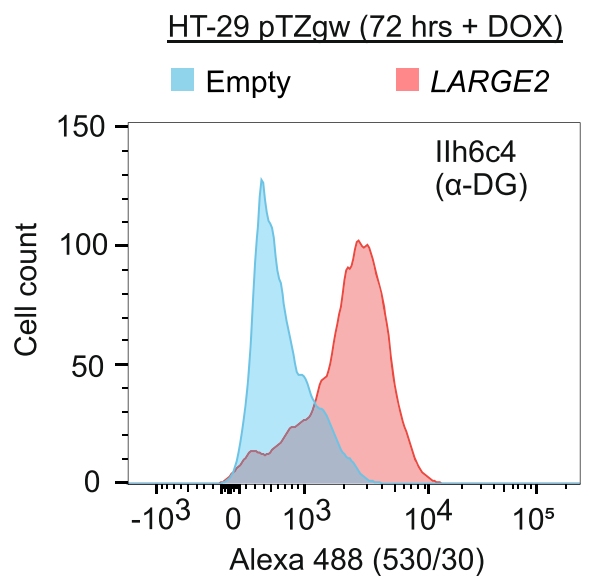

Fig. 3 (See legend on next page.) 
(See figure on previous page.)

Fig. 3 LARGE2 mediates functional O-glycosylation of a-DG in CRC. A) Upper panel: LARGE2 exons indicated as blue bars. GuideRNA 1 and 2 ( $\mathrm{g} 1$, g2) target sites in black, TCF7L2 binding site in yellow, start of coding sequence (CDS start) in Exon2 and STOP in Exon 14 (both in red). Lower panel: Mutation detection assay on genomic DNA from SW480 cells edited via CRISPR/Cas9 to achieve LARGE2 KO (g1, g2) or control (Ctrl). M: Marker. B) KO of LARGE2 by CRISPR/Cas9 in SW480 and SW620 cells (left panel) and PDTO1 (right panel) using g1 or g2. Western Blot analysis of WGA-enriched glycoproteins was used to detect a-DG, WCL were used to detect $\beta$-DG and tubulin. C) Immunoblot analysis and laminin overlay (Laminin-OL) of WGA-enriched glycoproteins after KO of LARGE2 via CRISPR/Cas9 in LS174T cells. $\beta$-DG and tubulin were analyzed on WCLs. D) LC-MS/MS analysis on HT-29 control (empty) or LARGE2 over-expressing cells. Cell lysates enriched for glycoproteins were run on SDS-PAGE and divided into 6 MW fractions B1-B6. Proteins of interest (DAG1, a-DG, LARGE2, and LAMA2) and their iBAQ (Intensity Based Absolute Quantification) are shown. E) Immunoblot analysis and laminin overlay (Laminin-OL) of WGA-enriched glycoproteins upon conditional ectopic expression of LARGE2 in HT-29 cells (500 ng/ml DOX for $72 \mathrm{~h}$ ). WCL were used to detect $\beta$-DG and tubulin. F) Flow cytometry analysis on control infected HT-29 cells (Empty, blue profile) or LARGE2 over-expressing cells (red profile) after treatment with DOX for $72 \mathrm{~h}$. Cells were stained with Ilh6c4 antibody and anti-mouse Alexa 488 secondary antibody. See Additional file 6 E for secondary antibody control

which, upon LARGE2-mediated O-glycosylation of $\alpha$ DG, co-precipitates with the WGA- $\alpha$-DG complex. Notably, only $\alpha$-DG-specific peptides consistently shifted over two MW windows with high intensity upon LARGE2 overexpression, and GPC4 was overall undetectable in HT-29 cells. This points to $\alpha$-DG as the main substrate of LARGE2 in this cellular background and verified our findings from immunoblot analysis using the O-glycosylation sensitive antibody IIh6 and a laminin-overlay assay on WGA-AE purified protein fractions (Fig. 3E). Furthermore, elevated cell surface abundance of O-glycosylated $\alpha$-DG upon ectopic LARGE2 expression was visualized by flow cytometry-assisted cell sorting (FACS) analysis after live cell immunolabelling (Fig. 3F, Additional file 6E). Our findings suggest that LARGE2 is both essential and sufficient for the attachment of laminin-binding matriglycan on $\alpha$-DG in CRC.

\section{Wnt signaling modulates functional O-glycosylation of a- DG via induction of LARGE2 in CRC}

We next asked whether modulation of Wnt signaling would affect LARGE2-dependent functional Oglycosylation of $\alpha$-DG. Indeed, shRNA-mediated silencing of APC or conditional ectopic expression of oncogenic CTNNB1-S33Y in HT-29 cells increased $\alpha$-DG Oglycosylation (Fig. 4A-C) and laminin binding capacity on the overlay assay (Fig. 4B,C). Importantly, LARGE2 mRNA induction and the increased $\mathrm{MW}$ matriglycan formation on $\alpha$-DG upon $A P C$ silencing depended on the integrity of the endogenous TCF7L2-BS within intron 1 of LARGE2 in CRISPR/Cas9-edited HT-29 cell lines (Fig. 4D,E, see Additional file $6 \mathrm{~F}$ for Sanger sequencing results on the mutated TCF7L2-BS). In accordance, interfering with $\beta$ catenin/TCF functionality in SW480-NE and LS174T-NE led to reduced levels of O-glycosylated $\alpha$-DG when compared to ERT2 control cells (Fig. 4F,G). DAG1 mRNA and $\beta$-DG protein did not significantly change upon Wnt modulation in CRC cells (Fig. 4A-G, Additional file 6G, $\mathrm{H})$. Furthermore, CRISPR/eCas9-mediated targeting of the TCF7L2-BS within LARGE2 in SW480 and in the CRC organoid line PDTO1 (Additional file 5), which led to decreased LARGE2 mRNA levels (Fig. 1I and Additional file 6I), compromised O-glycosylation of $\alpha$-DG (Fig. 4H,I). Taken together, these data suggest that Wnt signaling regulates the functional O-glycosylation of $\alpha$-DG in CRC cells, and this process depends on Wnt/TCF7L2mediated induction of $L A R G E 2$ gene expression.

\section{LARGE2 gene expression and a-DG 0-glycosylation are enriched in the Wnt-driven stem/progenitor compartment of human colonic epithelium}

To analyze the status of LARGE2 and $\alpha$-DG in selfrenewing hCoSCs, which depend on active Wnt signaling $[56,57]$, we isolated human colonic crypts from fresh tissue specimen and propagated them as patient-derived hCoSC-enriched organoids (PDOs) in a 3-dimensional (3-D) matrix (Matrigel ${ }^{\circ}$ ) overlaid with self-renewal promoting WREN culture media (Wnt3a, R-Spo3, EGF, Noggin), similar to what has been described previously (Fig. 5A) [2, 23]. Indeed, PDOs displayed expression of LARGE2 (qRT-PCR: Ct values 24-25 versus 32-33 for LARGE1) and, as visualized by immunoblotting, Ilh6c4reactive O-glycosylated $\alpha$-DG at a MW of $\sim 130-140$ $\mathrm{kDa}$ (Fig. 5B,C, Additional file 8A-C). This MW of $\alpha$-DG was lower than what we had observed in LS174T, SW480/620, and PDTO1 (Fig. 3B,C). Transfer of PDOs to culture medium devoid of Wnt and R-Spondin factors (EN medium), which triggers multi-lineage differentiation [2, 23], strongly decreased LARGE2 mRNA levels, similar to expression of bona-fide ISC markers LGR5 [38] and SMOC2 [58] (Fig. 5B, Additional file 8A,B). As expected, expression levels of KRT20 (pan-differentiation), ANPEP (enterocytes), TFF3 (goblet cells), and CHGA (enteroendocrine cells) were increased (Fig. 5B, Additional file 8A,B). In contrast to LARGE2, LARGE1 and DAG1 mRNA levels did not change during PDO differentiation (Fig. 5B, Additional file 8A,B). Importantly, differentiation of PDOs, indicated by lower levels of the hCoSC marker PTK7 [34], abolished $\alpha$-DG Oglycosylation (Fig. 5C, Additional file 8C). Interestingly, we also observed a reduction of $\beta$-DG protein upon PDO differentiation (5C, Additional file 8C). 


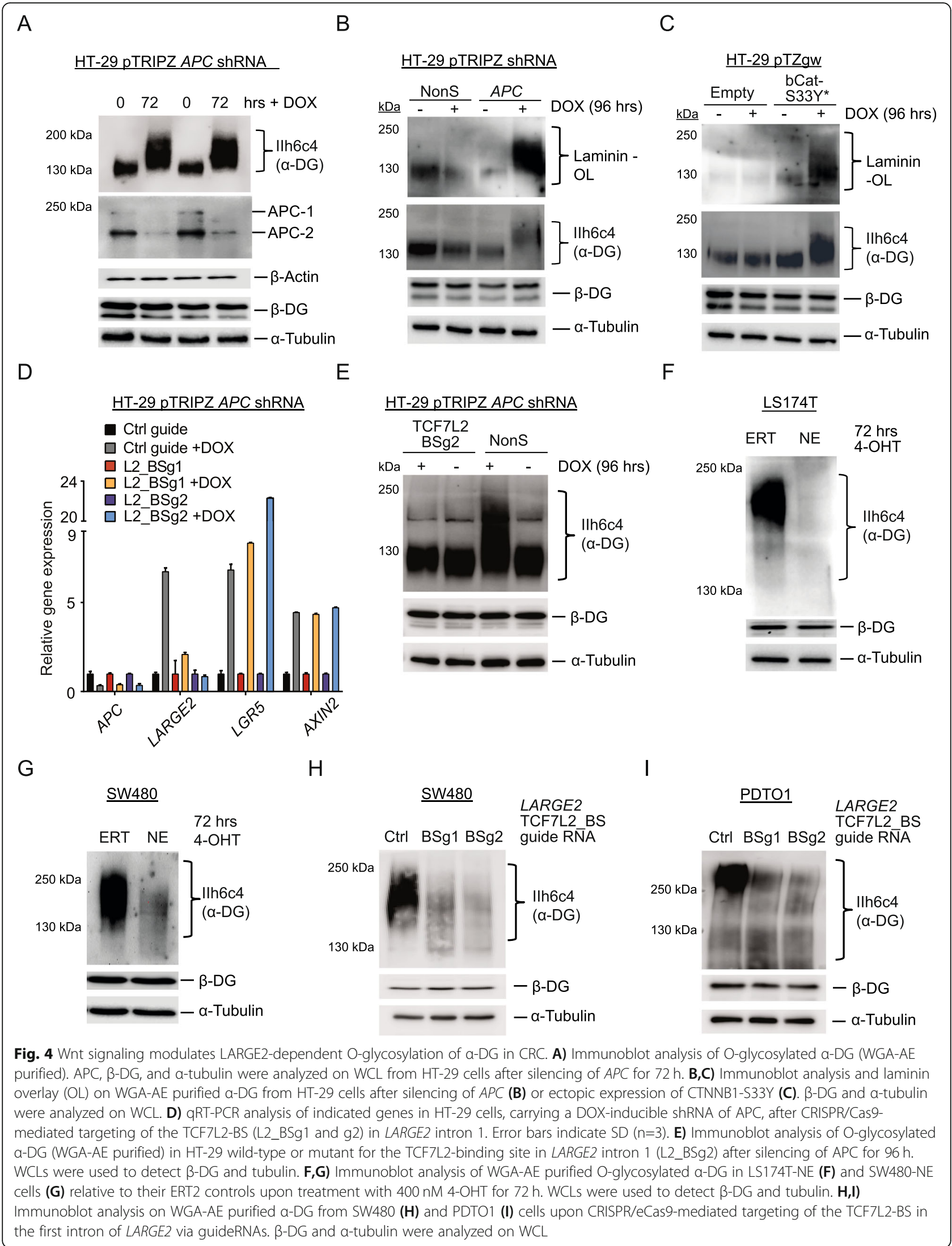


A

Human colonic organoids WREN media

Stem/progenitor cell-enriched

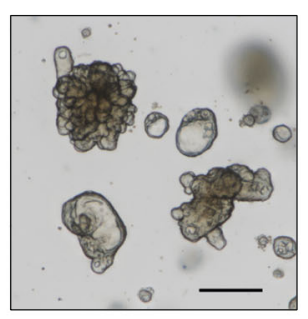

C

PDO1

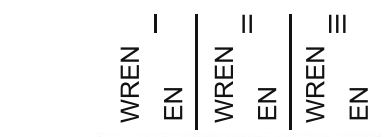

$130 \mathrm{kDa}$ ‥ $6 \mathrm{~km}+\}_{(\alpha-D G)}^{\mathrm{llh} 6 \mathrm{c} 4}$

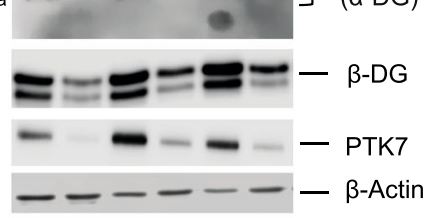

F

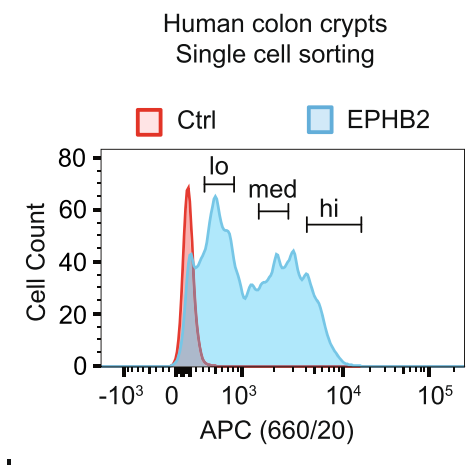

G
B

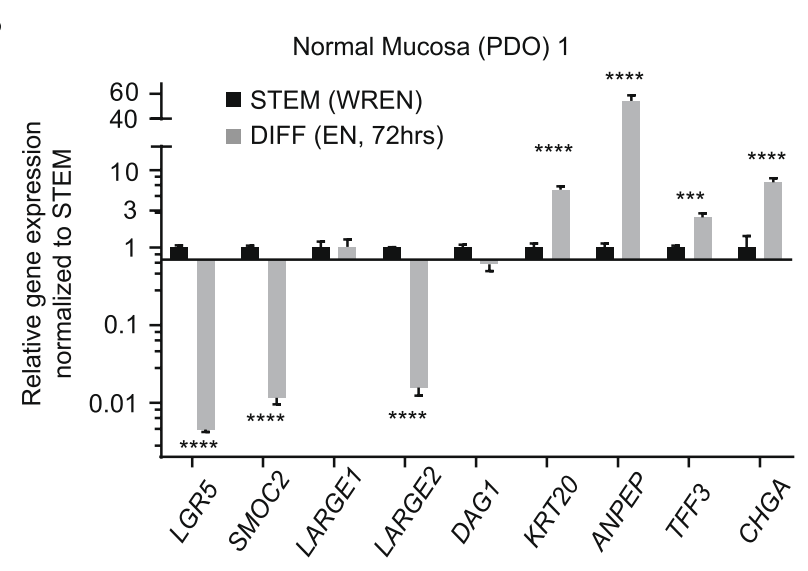

D PDO1 (WREN media) Single cell sorting

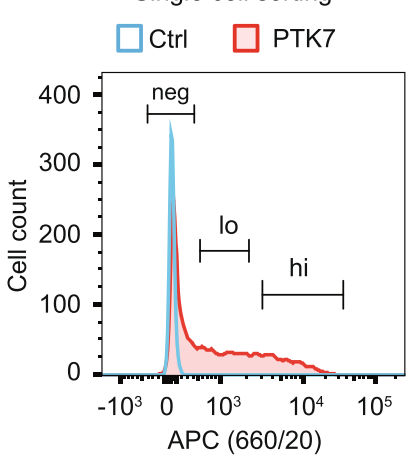

$$
\begin{aligned}
& \text { Human colon crypts } \\
& \text { Single cell sorting }
\end{aligned}
$$

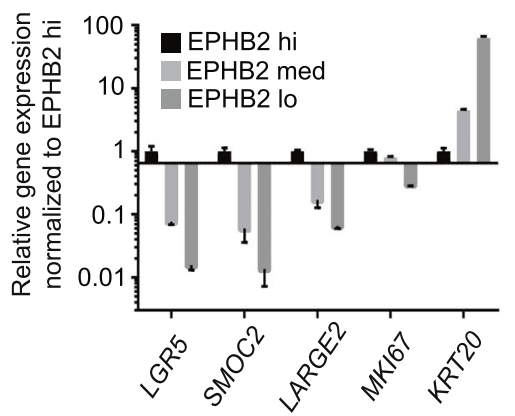

E PDO1 (WREN media) Single cell sorting

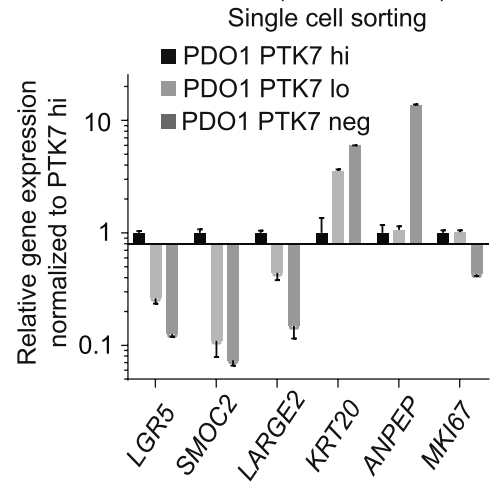

$\mathrm{H}$
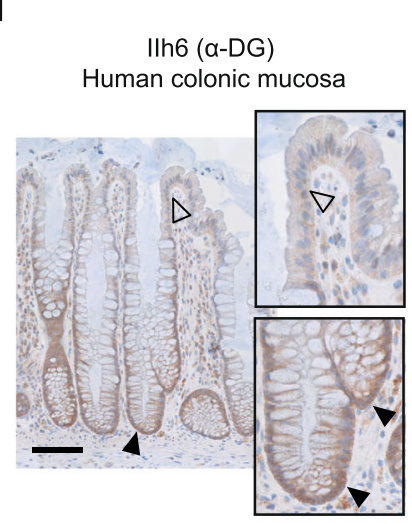

$\mathrm{K}$

Ilh6-IHC (a-DG), (mouse ileum)

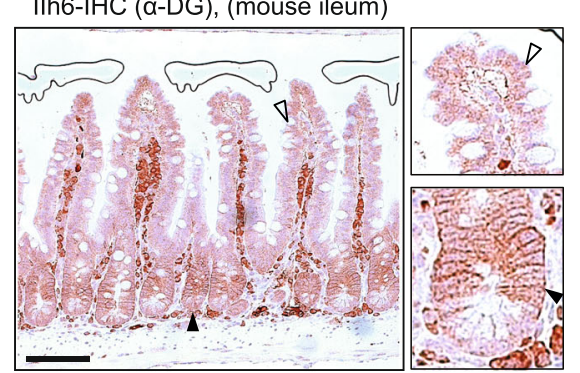

Fig. 5 (See legend on next page.) 
(See figure on previous page.)

Fig. 5 LARGE2 expression of a-DG O-glycosylation are enriched in the Wnt-driven stem/progenitor compartment of human colon epithelium. A) Human colonic organoids (PDOs) embedded in Matrigel and maintained in WREN (Wnt, R-Spondin, EGF, Noggin) medium. Scale bar represents $20 \mu \mathrm{m}$. B) qRT-PCR analysis of the indicated genes in PDO1 maintained in WREN (=STEM) or differentiation medium (DIFF, EN). Results are shown as mean $\pm \mathrm{SD}(n=3) .{ }^{* * *} p<0.001 ;{ }^{* * * *} p<0.0001$. See Additional file 8A,B for experiments on PDO2 and PDO3. C) Immunoblot analysis of a-DG from WGA-AE purified glycoproteins of PDO1, cultivated in WREN or EN media for $72 \mathrm{~h}$. WCL were used for analysis of $\beta$-DG, PTK7, and $\beta$-actin. D-G) FACS profile and Taqman ${ }^{\text {TM }}$ qRT-PCR analysis from PDO single cells -stained with APC-coupled antibody for PTK7 (D,E) or from human crypt epithelial cells, stained against EPHB2 (F,G). Control staining for viable cells (DAPI) was performed to define the PTK7 or EPHB2-negative fraction (Ctrl). In D) neg: PTK7 negative, lo: PTK7 low, hi: PTK7 high. In F) hi: EPHB2 high, med: EPHB2 medium, low: EPHB2 low cell fraction. Error bars in $\mathbf{E}$ and $\mathbf{G}$ indicate mean \pm SD ( $n=3$ technical replicates). See Additional file 8D,E for data on PDO2 and PDO3. H,I) Immunohistochemistry (IHC) analysis of a-DG (Illh6 antibody) on FFPE human colonic (H) or rectal (I) mucosa. Black arrowheads: crypt base specific staining; clear arrowheads: fading or loss of staining. Scale bars represent $100 \mu \mathrm{m}(\mathbf{H})$ and $50 \mu \mathrm{m}(\mathbf{I})$. J, K) In situ hybridization (ISH) with a Large2-specific probe (J) and IHC staining of glycosylated a-DG (K) on mouse small intestinal FFPE tissue (lleum). Scale Bar represents $100 \mu \mathrm{m}$. Black arrowheads: staining in crypts; clear arrowheads: lack of staining in villi. See Additional File 9 for control staining (no probe) and additional data

As has been reported previously, human PDOs maintained in WREN medium display a heterogeneity of stem and progenitor cells specified by relatively high or low surface abundance of the hCoSC marker PTK7 [34]. To examine LARGE2 expression in these PDO subpopulations, we performed FACS sorting to isolate PTK7high, PTK7-low, and PTK7-negative cells from PDOs derived from three different individuals: Strongest LARGE2 gene expression occurred in PTK7-high cells, which also showed higher levels of $L G R 5$ and SMOC2 mRNA when compared to PDO cells low or negative for PTK7 (Fig. 5D, E, Additional file 8D,E). Lowest LARGE2 expression was detected in PTK7-negative PDO cells characterized by relatively highest levels of the differentiation markers KRT2O and ANPEP (Fig. 5E, Additional file 8D,E). We received a similar result after sub-fractionation of primary mucosal tissue-derived crypt epithelial cells by FACS according to the surface abundance of EPHB2, which is highest in hCoSCs located at the crypt bottom [2] (Fig. 5 F). Here, expression of $L A R G E 2, L G R 5$ and $S M O C 2$ was most pronounced in the stem cell-enriched EPHB2-high fraction and stepwise declined in EPHB2-medium and EPHB2-low cells which represent transient amplifying cells at different stages of differentiation [2] (Fig. 5G). As expected, KRT2O levels showed an inverse gradient with highest expression in the EPHB2-low cell fraction (Fig. 5G). Next, we performed immunohistochemical (IHC) staining on formalin-fixed paraffin-embedded (FFPE) human colonic and rectal tissue for O-glycosylated $\alpha$-DG with the glycosylation-sensitive antibody IIh6. IHC staining was optimized on FFPE sections of human heart muscle tissue (Additional file 8F,G). By applying this protocol, we observed strongest immuno-reactivity against O-glycosylated $\alpha$-DG at the membrane of stem/progenitor cells of human colonic and rectal crypts while differentiated upper crypt cells did not show this feature (Fig. 5H,I). These data suggest that LARGE2/ $\alpha-D G$ signaling is differentiation-dependent in human colonic epithelium and mainly occurs in the Wnt-driven stem/progenitor cell compartment.

\section{A differentiation-dependent LARGE2/a-DG axis is conserved in mouse small intestinal epithelium}

To address if the differentiation dependent expression of LARGE2 observed in human PDOs was conserved among species, we performed in situ hybridization (ISH) with a Large2-specific probe on FFPE sections of mouse intestinal (ileum) tissue. Mild expression of Large2 mRNA mainly occurred at the bottom two-thirds of intestinal crypts, while intestinal villi, which harbor terminally differentiated cells, did not show expression of Large2 (Fig. 5), Additional file 9A). We observed a similar gradient of membranous O-glycosylated $\alpha$-DG by performing IHC on a serial FFPE section (Fig. 5K, see Additional file 9B for antibody controls on mouse tissue). Therefore, Large2 mRNA levels and O-glycosylation of $\alpha$-DG correlate with a non-differentiated status of mouse small intestinal epithelial cells.

\section{LARGE2 expression and O-glycosylation of a-DG depend on $A P C$ functionality in human adenoma organoids}

According to public microarray data derived from 32 patient-matched human colonic mucosa-adenoma pairs (GSE8671) [59], LARGE2 levels were slightly elevated in human adenoma specimen (Fig. 6A). ISH against Large2 on FFPE sections derived from ApcMin mice [60] revealed elevated abundance of Large 2 mRNA in adenoma (Fig. $6 \mathrm{~B}$, Additional file $9 \mathrm{C}, \mathrm{D})$, which also showed membranous $\mathrm{O}$-glycosylated $\alpha-\mathrm{DG}$ as visualized by IHC staining (Fig. $6 \mathrm{C})$. In human adenoma, we detected basal and membranous $\alpha$-DG via IHC staining on FFPE sections of tubular and tubular-villous adenomas which are typically driven by an intrinsically activated Wnt program (Fig. 6D). Colonic crypts in the vicinity of the dysplastic tissue displayed the expected restriction of O-glycosylated $\alpha$-DG to the stem/progenitor cell compartment at the crypt bottom (Fig. 6D, lower panel).

We next asked whether different truncation mutations of $A P C$ would differentially affect the level of LARGE2 and O-glycosylated $\alpha$-DG. Therefore, we performed CRISPR/Cas9-mediated genome editing on two benign 


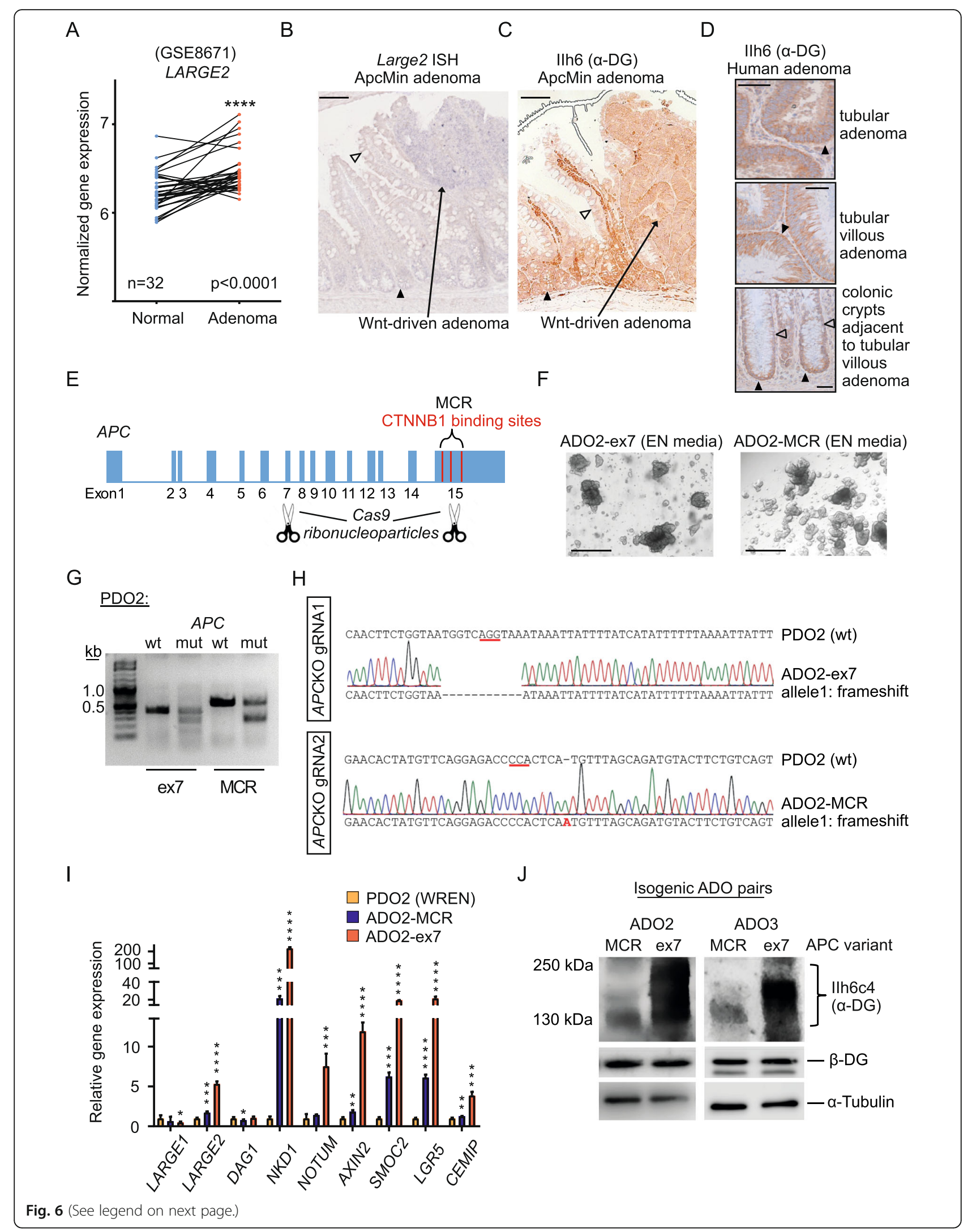


(See figure on previous page.)

Fig. 6 LARGE2 expression and a-DG O-glycosylation in engineered human adenoma organoids depends on the functionality of truncated APC. A) LARGE2 gene expression in a human dataset GSE8671 $(n=32)$, comparing normal human mucosa to matched adenoma tissue (*** $p<0.0001)$. B) ISH analysis using a Large2-specific probe on APCmin mouse FFPE adenoma tissue and adjacent intestinal crypts/villi (ileum). Black arrowhead: staining in normal crypts. Clear arrowheads: absent staining. Scale bar represents $100 \mu \mathrm{m}$. See Additional file $9 E$ for control. C,D) $1 H C$ staining for a-DG (Illh6 antibody) on Wnt-driven adenoma tissue of an APCmin mouse (C) or on human adenoma and adjacent normal tissue (D). Black arrowheads: membranous staining in crypt and adenoma regions, clear arrowheads: absent staining in upper crypts. Scale bar represents 100 (C) and $20 \mu \mathrm{m}$ (D). E,F) APC gene structure, CTNNB1 binding sites (20AAR sequences) in Exon 15. Two Cas9 ribonucleoparticles were assembled to target APC of PDOs in either Exon 7 (ex7) or 15 (MCR) (E), which yielded two isogenic adenoma organoid lines (ADO-ex7 and ADO-MCR) maintained in EN media (F). Scale bars represent $500 \mu \mathrm{m}$. G, H) Mutation detection assay $(\mathbf{G})$ and Sanger sequencing $(\mathbf{H})$ on genomic DNA derived from human normal mucosa (PDO2) organoids and isogenic adenoma organoid lines (ADO-ex7 and ADO-MCR). I) qRT-PCR analysis of the indicated genes from normal colon organoids (PDO2, in WREN media) and the isogenic ADO lines (ADO2-MCR and ADO2-ex7). Gene expression is relative to PDO2. Samples derived from one patient were analyzed three times. The results are shown as mean $\pm \mathrm{SD}$. ${ }^{* *} p<0.01 ;{ }^{* * *}$ $p<0.001 ;{ }^{* * * *} p<0.0001$. See Additional File 9F-H for data on PDO3. J) Immunoblot analysis of glycosylated a-DG on WGA-AE purified glycoproteins from two isogenic adenoma organoid pairs ADO2 and ADO3 (ex7 versus MCR) WCL was used for detection of DAG1, $\beta$-DG, and tubulin

PDO lines to introduce truncation mutations either in the MCR (Exon 15, guide RNA as described in [33]) or in Exon 7/8 (guide RNA as described in [61]) of $A P C$ (Fig. $6 \mathrm{E})$. As a selective measure, engineered human adenoma organoid lines (ADOs) were propagated in culture media lacking Wnt and RSPO factors (EN media) (Fig. 6F). Genome editing was confirmed by a mismatch cleavage assay and by Sanger sequencing (Fig. 6G,H, Additional file 9F, G). In those ADOs where $A P C$ had been mutated in the MCR (ADOs-MCR), so as to maintain 2*20AAR repeats in APC, expression of known Wnt targets was markedly lower when compared to ADOs carrying the short APC variant (ADOs-ex7) (Fig. 6I, Additional file 9H). Importantly, the $A P C$ exon7 truncation mutation caused a more than 3-fold higher expression of $L A R G E 2$ when compared to ADOs-MCR, while expression levels of $L A R G E 1$ and $D A G 1$ were equal in both types of ADOs (Fig. 6I, Additional file $9 \mathrm{H}$ ). Importantly, elevated levels of $L A R G E 2$ in ADOs-ex7 were associated with higher MW matriglycan structures on $\alpha$-DG (between 130 and $250 \mathrm{kDa}$ ) when compared to their APC-MCR mutant but otherwise isogenic counterparts $(\sim 130-150 \mathrm{kDa})$ (Fig. 6J), or PDOs ( 130-150 kDa, Fig. 5C), while $\beta$-DG levels were equal in the two ADO types (Fig. 6J). These data show that complete functional ablation of APC leads to aberrantly activated Wnt/LARGE2/ $\alpha$-DG signaling in human premalignant ADOs.

\section{LARGE2 gene expression is overall elevated in full-blown primary and liver metastatic CRC}

Examination of LARGE2 gene expression data derived from The Cancer Genome Atlas (TCGA, COAD and READ cohorts) [28, 46] revealed markedly elevated LARGE2 mRNA levels in advanced CRC when compared to normal tissue (Fig. 7A, Additional file 10A). To validate these data on patient-derived, purely epithelial CRC cells, we analyzed $L A R G E 2$ expression in 10 PDTO lines (Additional file 5). Importantly, all analyzed PDTOs were diagnosed as microsatellite stable and did not depend on Wnt-activating growth factors for long-term culture ( $>2$ month), indicative of cell-intrinsically activated Wnt signaling. 6 PDTOs were established from primary tumor tissue and 4 PDTOs from already liver metastasized CRC (see Additional file 5). As an approximation for non-differentiated human colonic epithelia, $L A R G E 2$ gene expression was determined in three PDO lines. In accordance with TCGA data, LARGE2 mRNA levels were heterogeneously elevated in PDTOs relative to PDOs $(\sim 2$ - to 25 -fold), which at least in part might be due to different levels of intrinsic Wnt activity in PDTOs (Fig. 7B). To better address if LARGE2 was differentially expressed between non-metastatic (M0) or liver-metastatic (M1) primary CRC tissues, we isolated total RNA from FFPE tumor-enriched areas from M0 $(n=12)$ or M1 $(n=12)$ CRC cases (Additional file 11). Analyses of these samples by $\operatorname{TaqMan}^{\mathrm{TM}}$ qRT-PCR assays showed a weak but non-significant tendency towards elevated LARGE2 in M1 CRC ( $p=0.083)$ (Fig. 7C). Similarly, RNA-Seq data derived from the TCGA-COAD cohort [46] did not show differential expression of LARGE2 between M0 $(n=365)$ and M1 $(n=71)$ tumors (Additional file 10), suggesting that the elevated LARGE2 gene expression level found in CRC cohorts and PDTOs is not affected by metastatic disease progression.

\section{a-DG O-glycosylation is heterogeneous in a panel of CRC organoids}

To address the status of O-glycosylated $\alpha-D G$ in our PDTO CRC panel, we performed immunoblot analysis on WGA-AE purified protein fractions: 7 out of 9 PDTOs showed clear IIh6c4 reactivity, the MW of Oglycosylated $\alpha-D G$ varied between $\sim 130-140 \mathrm{kDa}$ (PDTO2) and $\sim 250 \mathrm{kDa}$ (PDTO1, PDTO17), and glycosylated $\alpha-D G$ was almost absent or low in PDTO4 and PDTO13, respectively (Fig. 7D, Additional file 10 E,F). IHC staining against O-glycosylated $\alpha$-DG on FFPE CRC tissues from which the PDTOs originated gave concordant results (Fig. 7E, Additional file 10G). Elevated 
A

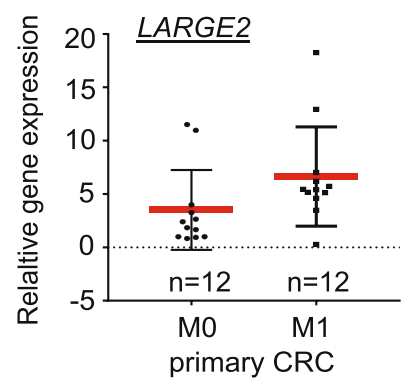

E

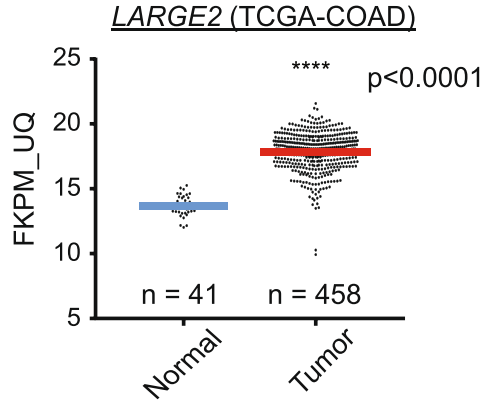

IIh6 ( $\alpha-D G)$ IHC staining
on primary tumor tissues
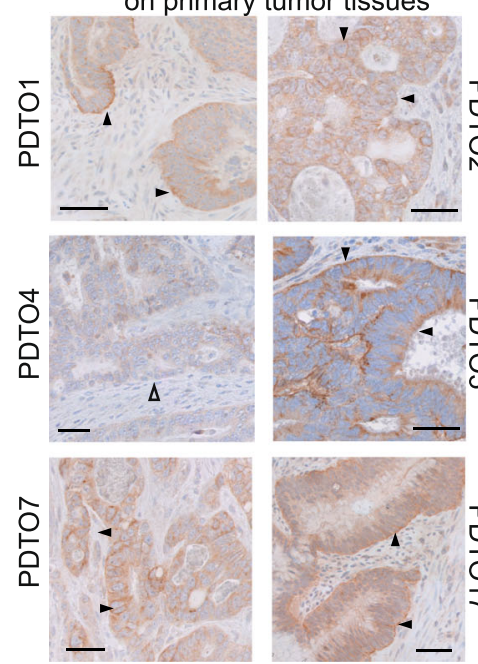

$\mathrm{H}$

KM12-L4a_luc on HMEC-1

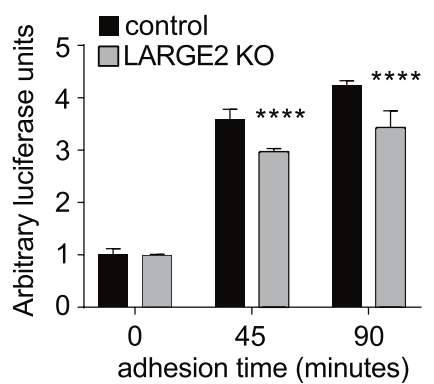

B

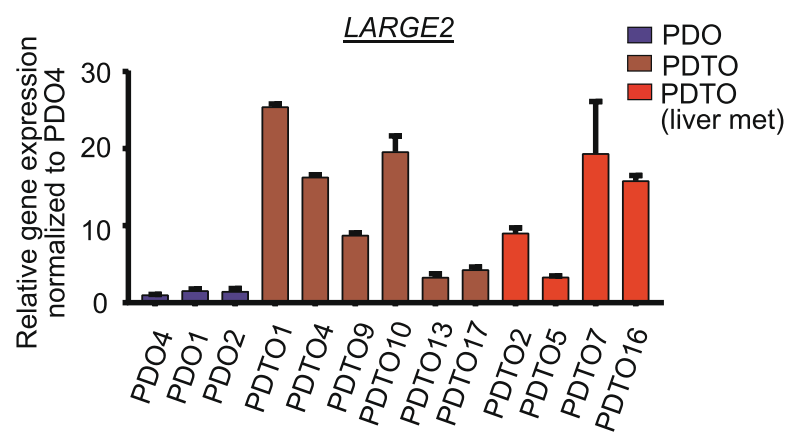

D

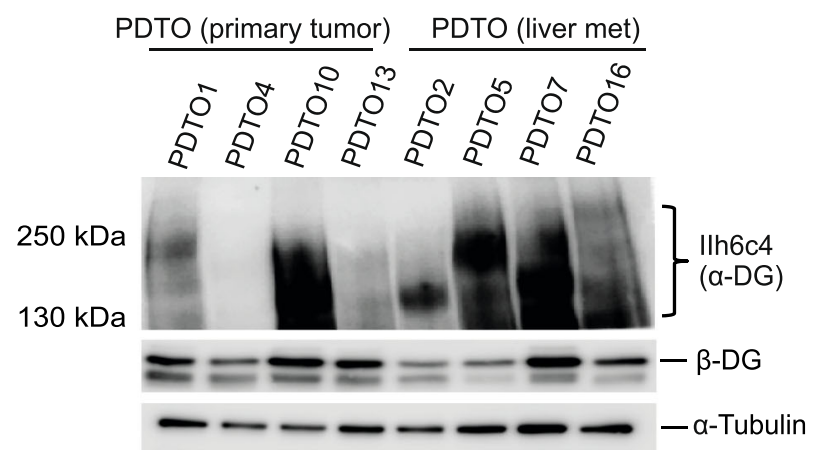

G

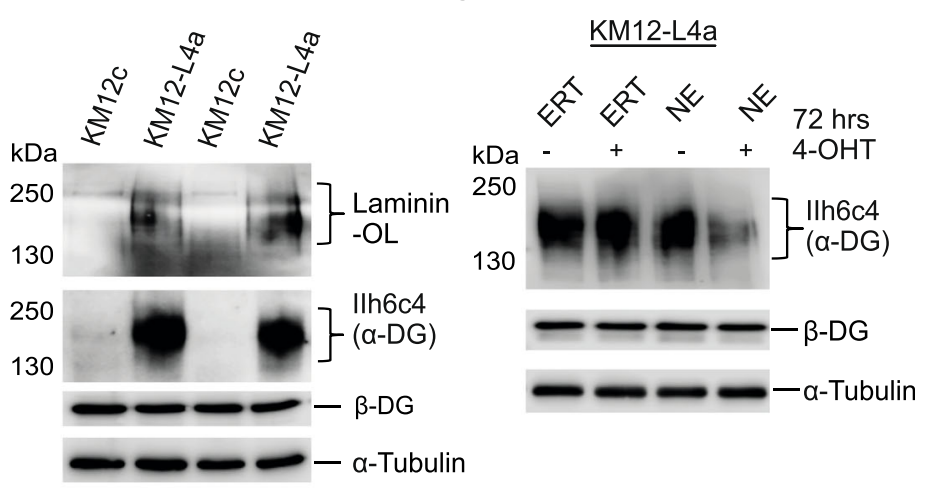

LS174T - LARGE2 KO (stably transduced pools)

HT-29 pTZgw + 72 hrs DOX (stably transduced pools)
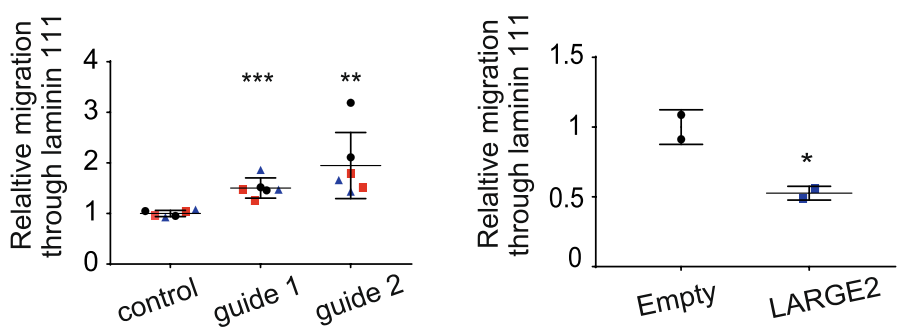

Fig. 7 (See legend on next page.) 
(See figure on previous page.)

Fig. 7 LARGE2/a-DG signaling is elevated in primary and liver metastatic CRC cells and affects CRC cell adhesion and migration. A) $L A R G E 2$ gene expression analysis on human TCGA-COAD dataset: comparison of normal human mucosa samples and tumor tissues. (*** $p<0.0001)$. B) qRTPCR analysis of LARGE2 in PDOs and PDTOs: colonic organoids (PDOs, blue), primary tumor organoids (PDTO, dark red), and liver metastasisderived tumor organoids (mPDTO, light red). Shown are mean values \pm SD ( $n=3$ technical replicates). C) TaqMan ${ }^{\mathrm{TM}}$ qRT-PCR analysis of LARGE2 expression on primary FFPE tissue samples comparing M0 and M1 CRC cases. D) Immunoblot analysis of glycosylated a-DG on WGA-AE purified glycoproteins from primary and metastatic (m)PDTOs. $\beta$-DG and a-Tubulin were detected on input WCL. E) IHC analysis of glycosylated a-DG (Illh6 antibody) on FFPE CRC tissues matching to the indicated PDTOs. Black arrowheads: specific staining. Clear arrowhead: negative staining. Scale bars: $50 \mu \mathrm{m}$. F,G) Immunoblot analysis of glycosylated a-DG and laminin overlay (Laminin-OL) on WGA-AE purified glycoproteins from KM12C and KM12-L4a cells (F) and from KM12-L4a-NE or -E cells non-treated or after treatment with $400 \mathrm{nM} 4-\mathrm{OHT}$ for $72 \mathrm{~h}(\mathbf{G}) . \beta-\mathrm{DG}$ and $\mathrm{a}-$ Tubulin were detected on WCL. H) Adhesion of KM12-L4a cells, stably transduced with a luciferase (luc)-encoding lentivirus and either wild-type or LARGE2 KO, was quantified at the indicated time points by measuring luc activity. $T=0$ represents the baseline control and was also used for sample normalization. The results are shown as mean $\pm S D(n=3)$, ${ }^{* * *} p<0.0001$. I,J) Transwell migration assays of CRC cells through laminin111 coated membranes. LS174T cell pools harboring a LARGE2 knockout (I), and HT-29 cells overexpressing LARGE2 (J) were compared to their control cell pools (*: $\left.p<0.05 ;{ }^{* *}: p<0.01 ;{ }^{* *}: p<0.001\right)$

LARGE2 gene expression in PDTO1, 10, and PDTO16 co-occurred with strong intensity and/or higher than "normal" MW versions of $\alpha$-DG as seen in PDOs (compare to Fig. 4), and PDTO13 showed relatively low levels of LARGE2 and O-glycosylated $\alpha$-DG. However, this correlation was not strictly given in all analyzed PDTO lines: PDTO2 and PDTO7 expressed high levels of LARGE2 but showed $\alpha-\mathrm{DG}$ at a comparable MW as seen in non-differentiated PDOs, and PDTO5 and PDTO17 displayed high MW $\alpha-D G$ variants in a background of mildly elevated $L A R G E 2$ levels (Fig. 7B,E, Additional file 10F). Therefore, it is likely that also other factors besides LARGE2 affect the complexity/MW of $\alpha$-DG-attached matriglycan in a subset of CRC. These data suggest that matriglycan formation on $\alpha$-DG represents a common feature of primary and liver-metastasized CRC cells.

\section{Wnt/LARGE-dependent O-glycosylation of a-DG affects adhesion to endothelial cells in liver metastatic CRC cells}

To address the status of O-glycosylated $\alpha$-DG in a wellcharacterized model of liver metastasis, we took advantage of the Fidler cell lines [62] KM12c, that shows a poor capability to colonize the liver after intrasplenic injection, and its liver metastatic derivative KM12-L4a. Here, we observed an enrichment of O-glycosylated $\alpha$ DG and laminin-binding capacity in KM12-L4a-derived glycoprotein fractions when compared to KM12c (Fig. 7F). This is in accordance with data from Frame M.C. and colleagues, who reported enhanced adhesiveness of KM12-L4a to laminin and other ECM-components relative to KM12c [63]. Importantly, CRISPR/Cas9-mediated $\mathrm{KO}$ of LARGE2 in KM12-L4a cells or acute blockade of Wnt signaling in KM12-L4a-NE, which down-modulated expression of LARGE2 as early as 24 $\mathrm{h}$, interfered with O-glycosylation of $\alpha$-DG (Fig. 7G, Additional file $10 \mathrm{H}, \mathrm{I})$. Interestingly, the adhesion of CRC cells to the laminin-rich ECM of the liver sinusoid has been demonstrated to play an important role in the formation of metastasis [64]. By performing an endothelial cell adhesion assay [65], we observed that the LARGE2 KO-mediated loss of laminin-binding $\alpha$-DG in KM12-L4a cells indeed interfered with their ability to rapidly attach to a confluent layer of HMEC-I immortalized human endothelial cells [66] (Fig. 7H). This might hint to a potential role of O-glycosylated $\alpha$-DG in the adhesion of circulating CRC cells to the laminin-rich ECM of blood vessels [67]. Future studies should therefore focus on the biological consequence of enhanced laminin-adhesiveness in the context of high Wnt/LARGE2/ $\alpha$-DG signaling for the liver colonization capacity of CRC cells.

\section{LARGE2/a-DG interferes with CRC cell migration through laminin-coated membranes}

Intriguingly, O-glycosylated $\alpha$-DG was described to interfere with cellular migration and invasiveness in renal and prostate cancer [68]. In accordance with this observation, we observed that LARGE2 KO-mediated loss of functional $\alpha$-DG in LS174T and SW620 cells (Fig. 2B,C) augmented in vitro cellular migration through laminin-coated membranes in a transwell migration assay (Fig. 7I and Additional file $10 \mathrm{~J}$ ). Accordingly, ectopic expression of LARGE2 in HT-29 cells, which led to the formation of high MW matriglycan on $\alpha-D G$ (Fig. 2E), interfered with cell migration in this setting (Fig. 7J). In agreement with what has been published previously for other tumor entities, our data point to a rather inhibitory role of the laminin adhesion-mediating Wnt/LARGE2/ $\alpha$-DG signaling pathway for cell migration and could therefore act as a limiting factor for the early dissemination of CRC cells from the primary tumor.

\section{Discussion}

Here, we describe a direct link between Wnt signaling and O-linked glycosylation in human colonic epithelial cells and in CRC. Extrinsic and intrinsic modulation of 
canonical Wnt activity triggered LARGE2 gene expression, and $L A R G E 2$ affected the abundance, complexity, and laminin-binding capacity of O-glycosylated $\alpha$-DG. As a component of the intestinal basement membrane (BM), laminin plays an important role in cell polarity, and different types of laminin are expressed in crypt and villus regions [69]. Recent efforts to adapt the human PDO model for growth in chemically defined designer matrices showed that laminin-111 (the major ingredient of Matrigel ${ }^{\circ}$ Matrix) affects ISC functionality [70]. Our finding that human colonic stem/progenitor cells feature a Wnt/LARGE2-dependent, laminin-binding matriglycan structure on $\alpha$-DG reveals a potentially relevant molecular trait of hCoSCs in this context. The upward migration of intestinal cells along the vertical crypt axis represents a tightly regulated process that involves epithelial cell-substratum interactions mediated by integrins, cytokines, and the ECM components laminin and collagen IV [71]. Notably, integrins and dystroglycan collaborate in myelin stabilization on peripheral nerves, and they play a redundant role during laminindependent epithelial polarization of epiblasts [72, 73]. Since the knock-out of $\beta$-integrin alone does not alter epithelial anchorage to the BM in the intestine of adult mice [74], several redundantly acting cell-matrix interactions might assure intestinal epithelial homeostasis. From our results, we suggest that also the Wnt/ LARGE2-dependent matriglycan structure on $\alpha$-DG might contribute to sustain the attachment of intestinal stem/progenitor cells to their laminin-rich BM.

Clinical data show that a large fraction of colorectal tumors with genetic alterations in $A P C$ express at least one truncated APC variant, which retains one to three 20AAR repeats able to bind and partially control $\beta$ catenin $[6,46,75]$. The consequence of this residual APC functionality is a sub-maximal, "just-right" dose of Wnt signaling supposed to optimally facilitate initial cellular transformation: Mice carrying $A P C$ alleles mutated within the MCR indeed display a more severe pre-malignant polyposis when compared to ApcMin mice expressing a shorter APC variant [76]. In accordance with these findings, we observed a slower proliferation phenotype in CRISPR/Cas9-engineered ADO-ex7 organoid lines when compared to their isogenic ADOMCR correspondents with lower intrinsic Wnt activity (data not shown). The stronger than "just-right" Wnt dose in pre-malignant ADO-ex7 organoids provoked elevated LARGE2 gene expression and the formation of "above-normal" molecular weight matriglycan structures on $\alpha$-DG $(>140 \mathrm{kDa})$, suggesting that LARGE2/ $\alpha$-DGmediated laminin adhesion might be oncogenic Wnt dose-dependent at this earliest, pre-malignant stage of CRC. Future studies should therefore clarify the consequences of aberrantly increased cellular adhesion to laminin for the initiation and progression of sporadic adenoma and polyposis.

We noticed that LARGE2 expression in full-blown $\mathrm{CRC}$ and patient-derived PDTOs was strongly elevated when compared to normal tissues and pre-malignant APC-MCR mutant ADOs, which might reflect an upregulation of tumor cell intrinsic Wnt activity during tumor progression. For instance, crosstalk with other oncogenic signaling pathways has been reported to potentiate Wnt signaling in CRC $[8,77]$. Alternatively, Wnt signalingindependent transcription factors might collaborate with the $\beta$-catenin/TCF7L2 complex in CRC to drive $L A R G E 2$ gene expression and $\alpha$-DG O-glycosylation beyond the levels found at pre-malignant disease stages. Nevertheless, CRISPR/Cas9-mediated mutagenesis of the endogenous TCF7L2 binding site in intron 1 of LARGE2 reduced $L A R G E 2$ mRNA levels and the occurrence of high MW matriglycan on $\alpha-D G$ in CRC cells and PDTOs. Together with our analyses on CRC cohorts, which showed a strong correlation of LARGE2 with the Wnt program, these data suggest that Wnt signaling represents the major driver of LARGE2-dependent matriglycan formation on $\alpha-D G$ in CRC.

While Wnt-driven CRC cells showed enhanced LARGE2 expression, matriglycan-complexity on $\alpha$-DG, and affinity towards laminin, studies on prostate and renal cancer provided evidence that O-glycosylated $\alpha$-DG interferes with cellular migration and invasiveness [21, 68]. We obtained concordant results from in vitro laminin migration assays when modulating the LARGE2/ $\alpha-D G$ axis. This points to Wnt/LARGE2/ $\alpha$-DG signaling as a rather tumor-restrictive mechanism at early invasive disease stages, where CRC cells need to overcome the boundaries of the BM to achieve tumor dissemination. Notably, accumulation of nuclear $\beta$-catenin in CRC represents a feature of so-called "migrating cancer stem cells" [16, 78]. Furthermore, Wnt signaling in CRC is potentiated by tumorassociated fibroblasts at the tumor stroma interface [9], and patients with tumors characterized by strong expression of the ISC gene signature are prone to suffer from disease recurrence [35]. Since $L A R G E 2$ was strongly associated with these pro-tumorigenic CRC traits according to our analyses, some highly Wnt-driven tumors might develop LARGE2-independent strategies in order to restrict or overcome a potentially migration-inhibitory effect of Wnt/LARGE2/ $\alpha$-DG signaling. As introduced, the physiological process of $\alpha$-DG O-glycosylation follows a complex order of events, and the action of multiple ( $>23$ ) enzymatic activities is necessary to create the glycosylation core on which LARGE1 or LARGE2 synthesize functional matriglycan. While we did not observe downregulation of either of these factors at the transcriptional level when analyzing NGS data derived from TCGA [46] (data not shown), approximately $18 \%$ of CRC cases (source: TCGA 
via cBioPortal, http://www.cbioportal.org) showed missense or truncating mutations in one or more of the respective genes (see Additional file 12). This might prevent or partially restrict matriglycan formation on $\alpha$-DG in the context of high Wnt activity and could explain the here observed heterogeneous pattern and different MW variants of O-glycosylated $\alpha$-DG in a panel of PDTOs. How distinct variants of $\mathrm{O}$-glycosylated $\alpha$-DG differentially affect the invasive behavior of CRC cells in a context of high Wnt activity needs to be further addressed.

Albeit our in vitro data point to a rather anti-cell migratory effect of Wnt/LARGE2/ $\alpha$-DG signaling, adhesion of CRC cells to ECM components of the liver sinusoid plays an important role in the formation of liver metastasis: E.g. therapeutic interference with integrin $\alpha 2-$ collagen IV or BCAM-laminin interactions prevented the liver metastatic spread of CRC cells in mouse models $[64,79]$. Indeed, we observed that several PDTOs derived from liver metastatic $\mathrm{CRC}$ had retained at least a stem/progenitor cell-like or an even elevated complexity of O-glycosylated $\alpha$-DG and a basal plus membranous localization of this laminin-binding feature. Together with our data on the liver-metastatic KM12c cell derivative KM12-L4a [62], which show that Wnt/LARGE2/ $\alpha$ DG signaling augments CRC cell adhesion to blood vessel endothelial cells, we speculate that CRC cell (sub)-populations highly positive for O-glycosylated $\alpha$ DG might get either selected for or simply become enriched due to their enhanced blood-vessel adhesiveness during liver colonization. From our in vitro functional data on Wnt/LARGE2/ $\alpha$-DG signaling, we can only speculate that the here identified Wnt/LARGE2/ $\alpha$ DG signaling pathway might play a dual role in CRC progression by limiting early tumor cell migration/dissemination while presumably facilitating liver colonization of intravasated, circulating CRC cells. However, future experiments using appropriate in vivo models that closely recapitulate the complete liver metastatic process are needed to clarify the biological relevance and a potentially dual role of the here identified Wnt/LARGE2/ $\alpha$-DG signaling pathway in metastatic CRC progression.

\section{Conclusions}

The expression of the LARGE2 O-glycosyltransferase encoding gene is regulated by physiologic, extrinsically stimulated Wnt signaling and also by an aberrantly activated Wnt pathway in the context of CRC cell intrinsic, Wnt-activating oncogenic mutations. Since localization of the Wnt transcription factor TCF7L2 to a canonical binding motif within the LARGE2 gene is necessary for Wnt-mediated LARGE2 mRNA induction, LARGE2 can be classified as a direct Wnt target gene.
LARGE2 levels are overall increased in full-blown CRC when compared to non-differentiated, benign colonic epithelial cells. In CRC, the expression of LARGE2 correlates with Wnt signaling intensity, an intestinal stem cell phenotype, and expression of human colonic epithelial stem cell genes in different cohorts of colon cancer patients.

According to our quantitative mass spectrometry data, $\alpha$-DG presumably represents the main target of LARGE2 in CRC, and our experimental data show that Wnt signaling mediates the functional $\mathrm{O}$-glycosylation of $\alpha$ DG and, as a biological consequence, the adhesion to laminin in a LARGE2-dependent manner.

LARGE2 gene expression and $\alpha$-DG O-glycosylation mainly occur in the Wnt-driven stem/progenitor cell compartment at the bottom of human colonic crypts. During CRC-initiation, the length and hence the functionality of truncated APC, which translates to different doses of oncogenic Wnt activity, influences the level of $L A R G E 2$ expression and the O-glycosylation status of $\alpha$ DG in human adenoma organoids.

Full blown CRC cells and patient-derived tumor organoids show an overall increased but heterogenous occurrence and molecular weight of O-glycosylated $\alpha$ DG, which at least partially depends on Wnt signaling and LARGE2 functionality.

Functionally, we found that LARGE/ $\alpha$-DG signaling inhibits CRC cell migration but also augments CRC cell adhesion to human blood vessel endothelial cells and might therefore play a context-dependent, dual role in CRC progression.

\section{Supplementary information}

Supplementary information accompanies this paper at https://doi.org/10. 1186/s12964-020-00561-6.

Additional file 1. Supplementary materials and methods.

Additional file 2. Wnt-signaling in CRC directly stimulates expression of LARGE2. Related to Fig. 1.

Additional file 3. Seq analysis after conditional silencing of APC in stably transduced HT-29 cells. Related to Fig. 1C.

Additional file 4. LARGE2 gene expression in CRC positively correlates with high Wht activity and elevated hCoSC gene expression. Related to Fig. 2.

Additional file 5. Informations on the patient-derived (tumor) organoid models used in this study.

Additional file 6. O-glycosylation of a-DG is mediated by Wnt-signaling through LARGE2. Related to Fig. 3 and Fig. 4.

Additional file 7. DAG1 peptide intensities from qLC-MS/MS analysis after overexpression of LARGE2 in HT-29 cells. Related to Fig. 3D.

Additional file 8. LARGE2 expression and O-glycosylation of a-DG in human PDOs and intestinal epithelium is enriched in the Wnt-driven stem/ progenitor cell compartment. Related to Fig. 5.

Additional file 9. $L A R G E 2$ expression in mouse adenoma and human engineered adenoma organoid (ADO) pairs carrying different APC truncation mutations. Related to Fig. 5J and Fig. 6. 
Additional file 10. $L A R G E 2 / a-D G$ expression in primary and liver metastatic CRC. Related to Fig. 7

Additional file $\mathbf{1 1}$ Information on the FFPE colorectal cancer tissue samples used for LARGE2 gene expression analysis. Related to Fig. 7C.

Additional file 12. Information on genetic status of several genes within the TCGA CRC cohort from TCGA PanCancer Atlas.

Additional file 13. List of used Primers, Oligonucleotides and Plasmids used in this study.

Additional file 14. Uncropped images of immunoblot membranes.

\section{Abbreviations}

CRC: Colorectal cancer; hCoSC: Human colonic stem cell; ISC: Intestinal stem cell; PDO: Patient-derived organoid; PDTO: Patient-derived tumor organoid; ECM: Extracellular matrix; APC: Adenomatous polyposis coli; $a-D G: ~ a-$ dystroglycan; qChIP: quantitative chromatin immuno-precipitation; WGA: Wheat germ agglutinin; BS: binding site; AE: Affinity enrichment; qRTPCR: Quantitative real-time PCR; CMS: Consensus molecular subtype; CRIS: Cancer cell intrinsic subtype; OL: Overlay; SD: Standard deviation; SEM: Standard error of the mean; FFPE: Formalin-fixed paraffin-embedded; MCR: Mutation cluster region; 4-OHT: 4-hydroxy-tamoxifen; EGF: Epidermal growth factor; DOX: Doxycycline; GW: Gateway; BM: Basement membrane

\section{Acknowledgements}

We thank Dr. Eduard Batlle (IRB Barcelona, Spain) for the FUW_nTCF7-ERT2 and FUW ERT2 lentiviral vectors used here to acutely block Wnt signaling in stably transduced CRC cell lines. We are also grateful to Prof. Dr. Isaiah Fidler (MD Anderson Cancer Center, Texas, USA) for KM12C and KM12-L4a cell lines, and Prof. Andreas Trumpp (HI-STEM at DKFZ Heidelberg) for the pV2Luc2 lentiviral vector. We further like to thank Andrea Sendelhofert and Anja Heier (LMU Munich) for support with immunohistochemical stainings, Prof. Dr. David Horst (Charité, Berlin), Dr. Markus Kaller and Dr. Matjaz Rokavec (LMU Munich) for advice with data analyses, and Dr. Stephanie Jaeckel and Dr. Markus Winter (LMU Munich) for reagents. We are grateful to Prof. Dr. Andreas Jung and Dr. Jörg Kumbrink (LMU, Munich) for molecular diagnostic analyses on PDTOs, and Dr. Moritz Jesinghaus (TUM, Munich) for support with PDO samples.

\section{Author contributions}

PJ conceptualized and supervised the project, designed and performed experiments, interpreted data, and wrote the manuscript. VD designed and performed experiments, analyzed data, and assisted with manuscript writing. CGD performed experiments on PDO/ADO/PDTOs, analyzed data, and assisted with manuscript design. SW and BK performed LC-MS/MS analyses and analyzed data. SB supported work on tumor organoids. MM provided RNA samples isolated from FFPE CRC material. HH interpreted data and provided material and access to instrumentation. JN provided tissue samples for PDOs and PDTOs, and FFPE tissue samples.

All authors read and approved the final manuscript.

\section{Authors' information}

Not applicable.

\section{Funding}

The project was funded by the German Cancer Consortium (DKTK), by the Ludwig-Maximilians-University (LMU) Munich, and by the Deutsche Forschungsgemeinschaft (DFG, GZ: JU 3072/1-1) (given to PJ).

\section{Availability of data and materials}

The datasets supporting the conclusions of this article are available in the following repositories:

- RNA-Seq data: Gene Expression Omnibus Accession-No:

GSE131575, https://www.ncbi.nlm.nih.gov/geo/query/acc.cgi?acc=GSE1315 75

- LC-MS/MS data: PRIDE database, project Accession-

No.: PXD013800, https://www.ebi.ac.uk/pride/

\section{Ethics approval and consent to participate}

Work with irreversibly anonymized human colon and CRC patient material has been approved by the ethical committee of the LMU Munich for our projects (project-No. 591-16-UE and 17-771-UE). FFPE tissue samples from the ApcMin mouse model were provided by the $\mathrm{HH}$ lab. Experimentation with these animals was approved by the Government of Upper Bavaria, Germany (AZ 55.2-1-54-2532-4-2014).

\section{Consent for publication}

Not applicable.

\section{Competing interests}

The authors declare that they have no competing financial interests.

\section{Author details}

${ }^{1}$ German Cancer Research Center (DKFZ), Heidelberg, Germany. ${ }^{2}$ German Cancer Consortium (DKTK), Partner site Munich, Munich, Germany. ${ }^{3}$ DKTK Research Group, Oncogenic Signaling Pathways of Colorectal Cancer, Institute of Pathology, Ludwig-Maximilians-Universität München, Munich, Germany. ${ }^{4}$ Chair of Proteomics and Bioanalytics, Technical University of Munich, Freising, Germany. ${ }^{5}$ Institute of Pathology,

Ludwig-Maximilians-Universität München, Munich, Germany. ${ }^{6}$ Bavarian Center for Biomolecular Mass Spectrometry, Freising, Germany. ${ }^{7}$ DKTK AG Oncogenic Signal Transduction Pathways in Colorectal/Pancreatic Cancer, Deutsches Krebsforschungszentrum (DKFZ) Heidelberg, DKTK Partnerstandort München, Institut für Pathologie der Ludwig-Maximilians-Universität (LMU) München, Thalkirchner Straße 36, D-80337 Munich, Germany.

Received: 17 December 2019 Accepted: 23 March 2020

Published online: 25 June 2020

\section{References}

1. van de Wetering $M$, Sancho E, Verweij $C$, de Lau W, Oving I, Hurlstone A, van der Horn K, Batlle E, Coudreuse D, Haramis AP, et al. The beta-catenin/ TCF-4 complex imposes a crypt progenitor phenotype on colorectal cancer cells. Cell. 2002;111:241-50.

2. Jung P, Sato T, Merlos-Suarez A, Barriga FM, Iglesias M, Rossell D, Auer H, Gallardo M, Blasco MA, Sancho E, et al. Isolation and in vitro expansion of human colonic stem cells. Nat Med. 2011;17:1225-7.

3. Zeineldin M, Neufeld KL. Understanding phenotypic variation in rodent models with germline Apc mutations. Cancer Res. 2013;73:2389-99.

4. Yang J, Zhang W, Evans PM, Chen X, He X, Liu C. Adenomatous polyposis coli (APC) differentially regulates beta-catenin phosphorylation and ubiquitination in colon cancer cells. J Biol Chem. 2006;281:17751-7.

5. Christie M, Jorissen RN, Mouradov D, Sakthianandeswaren A, Li S, Day F, Tsu C, Lipton L, Desai J, Jones IT, et al. Different APC genotypes in proximal and distal sporadic colorectal cancers suggest distinct WNT/beta-catenin signalling thresholds for tumourigenesis. Oncogene. 2013;32:4675-82.

6. Schell MJ, Yang M, Teer JK, Lo FY, Madan A, Coppola D, Monteiro AN, Nebozhyn MV, Yue B, Loboda A, et al. A multigene mutation classification of 468 colorectal cancers reveals a prognostic role for APC. Nat Commun. 2016;7:11743.

7. Blaj C, Schmidt EM, Lamprecht S, Hermeking H, Jung A, Kirchner T, Horst D. Oncogenic effects of high MAPK activity in colorectal Cancer mark progenitor cells and persist irrespective of RAS mutations. Cancer Res. 2017; 77:1763-74.

8. Ormanns S, Neumann J, Horst D, Kirchner T, Jung A. WNT signaling and distant metastasis in colon cancer through transcriptional activity of nuclear beta-catenin depend on active PI3K signaling. Oncotarget. 2014:5:2999-3011.

9. Vermeulen L, De Sousa EMF, van der Heijden M, Cameron $K$, de Jong JH, Borovski T, Tuynman JB, Todaro M, Merz C, Rodermond H, et al. Wnt activity defines colon cancer stem cells and is regulated by the microenvironment. Nat Cell Biol. 2010;12:468-76.

10. Wiese KE, Nusse R, van Amerongen R. Wnt signalling: conquering complexity. Development. 2018;145.

11. van Es JH, Haegebarth A, Kujala P, Itzkovitz S, Koo BK, Boj SF, Korving J, van den Born M, van Oudenaarden A, Robine S, Clevers H. A critical role for the Wnt effector Tcf4 in adult intestinal homeostatic self-renewal. Mol Cell Biol. 2012;32:1918-27.

12. Jamora C, Fuchs E. Intercellular adhesion, signalling and the cytoskeleton. Nat Cell Biol. 2002;4:E101-8. 
13. Smith KJ, Levy DB, Maupin P, Pollard TD, Vogelstein B, Kinzler KW. Wild-type but not mutant APC associates with the microtubule cytoskeleton. Cancer Res. 1994;54:3672-5.

14. Gradl D, Kuhl M, Wedlich D. The Wnt/Wg signal transducer beta-catenin controls fibronectin expression. Mol Cell Biol. 1999;19:5576-87.

15. Herbst A, Jurinovic V, Krebs S, Thieme SE, Blum H, Goke B, Kolligs FT. Comprehensive analysis of beta-catenin target genes in colorectal carcinoma cell lines with deregulated Wnt/beta-catenin signaling. BMC Genomics. 2014;15:74.

16. Basu S, Cheriyamundath S, Ben-Ze'ev A. Cell-cell adhesion: linking Wnt/betacatenin signaling with partial EMT and stemness traits in tumorigenesis. F1000Res 2018, 7.

17. Michele DE, Campbell KP. Dystrophin-glycoprotein complex: posttranslational processing and dystroglycan function. J Biol Chem. 2003;278: 15457-60.

18. Sheikh MO, Halmo SM, Wells L. Recent advancements in understanding mammalian O-mannosylation. Glycobiology. 2017;27:806-19.

19. Ashikov A, Buettner FF, Tiemann B, Gerardy-Schahn R, Bakker H. LARGE2 generates the same xylose- and glucuronic acid-containing glycan structures as LARGE. Glycobiology. 2013;23:303-9.

20. Yoshida-Moriguchi T, Yu L, Stalnaker SH, Davis S, Kunz S, Madson M, Oldstone MB, Schachter H, Wells L, Campbell KP. O-mannosyl phosphorylation of alpha-dystroglycan is required for laminin binding. Science. 2010;327:88-92.

21. Esser AK, Miller MR, Huang Q, Meier MM. Beltran-Valero de Bernabe D, Stipp CS, Campbell KP, lynch CF, Smith BJ, Cohen MB, Henry MD: loss of LARGE2 disrupts functional glycosylation of alpha-dystroglycan in prostate cancer. J Biol Chem. 2013;288:2132-42.

22. Fujimura K, Sawaki H, Sakai T, Hiruma T, Nakanishi N, Sato T, Ohkura T, Narimatsu H. LARGE2 facilitates the maturation of alpha-dystroglycan more effectively than LARGE. Biochem Biophys Res Commun. 2005;329:1162-71.

23. Sato $T$, Stange DE, Ferrante $M$, Vries RG, Van Es JH, Van den Brink $S$, Van Houdt WJ, Pronk A, Van Gorp J, Siersema PD, Clevers H. Long-term expansion of epithelial organoids from human colon, adenoma, adenocarcinoma, and Barrett's epithelium. Gastroenterology. 2011;141:1762-72.

24. Heinz S, Benner C, Spann N, Bertolino E, Lin YC, Laslo P, Cheng JX, Murre C, Singh $H$, Glass CK. Simple combinations of lineage-determining transcription factors prime cis-regulatory elements required for macrophage and B cell identities. Mol Cell. 2010;38:576-89.

25. Kim D, Pertea G, Trapnell C, Pimentel H, Kelley R, Salzberg SL. TopHat2: accurate alignment of transcriptomes in the presence of insertions, deletions and gene fusions. Genome Biol. 2013;14:R36.

26. Anders S, Pyl PT, Huber W. HTSeq--a Python framework to work with highthroughput sequencing data. Bioinformatics. 2015;31:166-9.

27. Love Ml, Huber W, Anders S. Moderated estimation of fold change and dispersion for RNA-seq data with DESeq2. Genome Biol. 2014;15:550

28. Grossman RL, Heath AP, Ferretti V, Varmus HE, Lowy DR, Kibbe WA, Staudt LM. Toward a shared vision for Cancer genomic data. N Engl J Med. 2016; 375:1109-12.

29. Marisa L, de Reynies A, Duval A, Selves J, Gaub MP, Vescovo L, EtienneGrimaldi MC, Schiappa R, Guenot D, Ayadi M, et al. Gene expression classification of colon cancer into molecular subtypes: characterization, validation, and prognostic value. PLoS Med. 2013;10:e1001453.

30. Isella C, Brundu F, Bellomo SE, Galimi F, Zanella E, Porporato R, Petti C, Fiori A, Orzan F, Senetta R, et al. Selective analysis of cancer-cell intrinsic transcriptional traits defines novel clinically relevant subtypes of colorectal cancer. Nat Commun. 2017:8:15107

31. Guinney J, Dienstmann R, Wang X, de Reynies A, Schlicker A, Soneson C, Marisa L, Roepman P, Nyamundanda G, Angelino P, et al. The consensus molecular subtypes of colorectal cancer. Nat Med. 2015;21:1350-6.

32. Subramanian A, Tamayo P, Mootha VK, Mukherjee S, Ebert BL, Gillette MA, Paulovich A, Pomeroy SL, Golub TR, Lander ES, Mesirov JP. Gene set enrichment analysis: a knowledge-based approach for interpreting genome-wide expression profiles. Proc Natl Acad Sci U S A. 2005;102: 15545-50.

33. Drost J, van Jaarsveld RH, Ponsioen B, Zimberlin C, van Boxtel R, Buijs A, Sachs N, Overmeer RM, Offerhaus GJ, Begthel H, et al. Sequential cancer mutations in cultured human intestinal stem cells. Nature. 2015; 521:43-7.

34. Jung P, Sommer C, Barriga FM, Buczacki SJ, Hernando-Momblona X, Sevillano M, Duran-Frigola M, Aloy P, Selbach M, Winton DJ, Batlle E.
Isolation of human Colon stem cells using surface expression of PTK7. Stem Cell Reports. 2015;5:979-87.

35. Merlos-Suarez A, Barriga FM, Jung P, Iglesias M, Cespedes MV, Rossell D, Sevillano M, Hernando-Momblona X, da Silva-Diz V, Munoz P, et al. The intestinal stem cell signature identifies colorectal cancer stem cells and predicts disease relapse. Cell Stem Cell. 2011;8:511-24.

36. Rosin-Arbesfeld R, Cliffe A, Brabletz T, Bienz M. Nuclear export of the APC tumour suppressor controls beta-catenin function in transcription. EMBO J. 2003;22:1101-13.

37. Jho EH, Zhang T, Domon C, Joo CK, Freund JN, Costantini F. Wnt/betacatenin/Tcf signaling induces the transcription of Axin2, a negative regulator of the signaling pathway. Mol Cell Biol. 2002;22:1172-83.

38. Barker N, van Es JH, Kuipers J, Kujala P, van den Born M, Cozijnsen M, Haegebarth A, Korving J, Begthel H, Peters PJ, Clevers H. Identification of stem cells in small intestine and colon by marker gene Lgr5. Nature. 2007; 449:1003-7

39. Hankey W, Frankel WL, Groden J. Functions of the APC tumor suppressor protein dependent and independent of canonical WNT signaling: implications for therapeutic targeting. Cancer Metastasis Rev. 2018;37:159-72.

40. Kolligs FT, Hu G, Dang CV, Fearon ER. Neoplastic transformation of RK3E by mutant beta-catenin requires deregulation of Tcf/Lef transcription but not activation of c-myc expression. Mol Cell Biol. 1999;19:5696-706.

41. Rosenbloom KR, Sloan CA, Malladi VS, Dreszer TR, Learned K, Kirkup VM, Wong MC, Maddren M, Fang R, Heitner SG, et al. ENCODE data in the UCSC genome browser: year 5 update. Nucleic Acids Res. 2013;41:D56-63.

42. van de Wetering M, Cavallo R, Dooijes D, van Beest M, van Es J, Loureiro J, Ypma A, Hursh D, Jones T, Bejsovec A, et al. Armadillo coactivates transcription driven by the product of the Drosophila segment polarity gene dTCF. Cell. 1997:88:789-99.

43. Yochum GS. Multiple Wnt/ss-catenin responsive enhancers align with the MYC promoter through long-range chromatin loops. PLoS One. 2011;6:e18966.

44. Slaymaker IM, Gao L, Zetsche B, Scott DA, Yan WX, Zhang F. Rationally engineered Cas9 nucleases with improved specificity. Science. 2016;351:84-8.

45. Korinek V, Barker N, Morin PJ, van Wichen D, de Weger R, Kinzler KW, Vogelstein B, Clevers $\mathrm{H}$. Constitutive transcriptional activation by a betacatenin-Tcf complex in APC-/- colon carcinoma. Science. 1997;275:1784-7.

46. Cancer Genome Atlas N. Comprehensive molecular characterization of human colon and rectal cancer. Nature. 2012:487:330-7.

47. Calon A, Lonardo E, Berenguer-Llergo A, Espinet E, Hernando-Momblona X, Iglesias M, Sevillano M, Palomo-Ponce S, Tauriello DV, Byrom D, et al. Stromal gene expression defines poor-prognosis subtypes in colorectal cancer. Nat Genet. 2015;47:320-9.

48. Isella C, Terrasi A, Bellomo SE, Petti C, Galatola G, Muratore A, Mellano A, Senetta R, Cassenti A, Sonetto C, et al. Stromal contribution to the colorectal cancer transcriptome. Nat Genet. 2015;47:312-9.

49. Liberzon A, Birger C, Thorvaldsdottir H, Ghandi M, Mesirov JP, Tamayo P. The molecular signatures database (MSigDB) hallmark gene set collection. Cell Syst. 2015;1:417-25.

50. Michels BE, Mosa MH, Grebbin BM, Yepes D, Darvishi T, Hausmann J, Urlaub H, Zeuzem S, Kvasnicka HM, Oellerich T, Farin HF. Human colon organoids reveal distinct physiologic and oncogenic Wnt responses. J Exp Med. 2019; 216:704-20

51. de Lau W, Barker N, Clevers H. WNT signaling in the normal intestine and colorectal cancer. Front Biosci. 2007;12:471-91.

52. Barretina J, Caponigro G, Stransky N, Venkatesan K, Margolin AA, Kim S, Wilson CJ, Lehar J, Kryukov GV, Sonkin D, et al. The Cancer cell line encyclopedia enables predictive modelling of anticancer drug sensitivity. Nature. 2012;483:603-7.

53. Scholer-Dahirel A, Schlabach MR, Loo A, Bagdasarian L, Meyer R, Guo R, Woolfenden S, Yu KK, Markovits J, Killary K, et al. Maintenance of adenomatous polyposis coli (APC)-mutant colorectal cancer is dependent on Wnt/betacatenin signaling. Proc Natl Acad Sci U S A. 2011;108:17135-40.

54. Heinen CD, Richardson D, White R, Groden J. Microsatellite instability in colorectal adenocarcinoma cell lines that have full-length adenomatous polyposis coli protein. Cancer Res. 1995;55:4797-9.

55. Inamori Kl, Beedle AM, de Bernabe DB, Wright ME, Campbell KP. LARGE2dependent glycosylation confers laminin-binding ability on proteoglycans. Glycobiology. 2016;26:1284-96.

56. Korinek V, Barker N, Moerer P, van Donselaar E, Huls G, Peters PJ, Clevers H. Depletion of epithelial stem-cell compartments in the small intestine of mice lacking Tcf-4. Nat Genet. 1998;19:379-83. 
57. Pinto D, Gregorieff A, Begthel $H$, Clevers $H$. Canonical Wnt signals are essential for homeostasis of the intestinal epithelium. Genes Dev. 2003;17:1709-13.

58. Munoz J, Stange DE, Schepers AG, van de Wetering M, Koo BK, Itzkovitz S, Volckmann R, Kung KS, Koster J, Radulescu S, et al. The Lgr5 intestinal stem cell signature: robust expression of proposed quiescent '+4' cell markers. EMBO J. 2012;31:3079-91.

59. Sabates-Bellver J, Van der Flier LG, de Palo M, Cattaneo E, Maake C, Rehrauer H, Laczko E, Kurowski MA, Bujnicki JM, Menigatti M, et al. Transcriptome profile of human colorectal adenomas. Mol Cancer Res. 2007;5:1263-75.

60. Moser AR, Pitot HC, Dove WF. A dominant mutation that predisposes to multiple intestinal neoplasia in the mouse. Science. 1990;247:322-4.

61. Matano M, Date S, Shimokawa M, Takano A, Fujii M, Ohta Y, Watanabe T, Kanai T, Sato T. Modeling colorectal cancer using CRISPR-Cas9-mediated engineering of human intestinal organoids. Nat Med. 2015;21:256-62.

62. Morikawa K, Walker SM, Nakajima M, Pathak S, Jessup JM, Fidler IJ. Influence of organ environment on the growth, selection, and metastasis of human colon carcinoma cells in nude mice. Cancer Res. 1988;48:6863-71.

63. Jones RJ, Avizienyte E, Wyke AW, Owens DW, Brunton VG, Frame MC. Elevated $\mathrm{c}$-Src is linked to altered cell-matrix adhesion rather than proliferation in KM12C human colorectal cancer cells. Br J Cancer. 2002;87:1 128-35.

64. Bartolini A, Cardaci S, Lamba S, Oddo D, Marchio C, Cassoni P, Amoreo CA, Corti G, Testori A, Bussolino F, et al. BCAM and LAMA5 mediate the recognition between tumor cells and the endothelium in the metastatic spreading of KRAS-mutant colorectal Cancer. Clin Cancer Res. 2016;22:4923-33.

65. Safuan S, Storr SJ, Patel PM, Martin SG. A comparative study of adhesion of melanoma and breast cancer cells to blood and lymphatic endothelium. Lymphat Res Biol. 2012;10:173-81.

66. Ades EW, Candal FJ, Swerlick RA, George VG, Summers S, Bosse DC, Lawley TJ. HMEC-1: establishment of an immortalized human microvascular endothelial cell line. J Invest Dermatol. 1992;99:683-90.

67. Yousif LF, Di Russo J, Sorokin L. Laminin isoforms in endothelial and perivascular basement membranes. Cell Adhes Migr. 2013;7:101-10.

68. Bao X, Kobayashi M, Hatakeyama S, Angata K, Gullberg D, Nakayama J, Fukuda MN, Fukuda M. Tumor suppressor function of laminin-binding alpha-dystroglycan requires a distinct beta3-N-acetylglucosaminyltransferase. Proc Natl Acad Sci U S A. 2009:106:12109-14.

69. Meran L, Baulies A, Li VSW. Intestinal stem cell niche: the extracellular matrix and cellular components. Stem Cells Int. 2017;2017:7970385.

70. Gjorevski N, Sachs N, Manfrin A, Giger S, Bragina ME, Ordonez-Moran P, Clevers H, Lutolf MP. Designer matrices for intestinal stem cell and organoid culture. Nature. 2016;539:560-4.

71. Patterson AM, Watson AJM. Deciphering the complex signaling systems that regulate intestinal epithelial cell death processes and shedding. Front Immunol. 2017;8:841.

72. Li S, Qi Y, McKee K, Liu J, Hsu J, Yurchenco PD. Integrin and dystroglycan compensate each other to mediate laminin-dependent basement membrane assembly and epiblast polarization. Matrix Biol. 2017;57-58:272-84.

73. Nodari A, Previtali SC, Dati G, Occhi S, Court FA, Colombelli C, Zambroni D, Dina G, Del Carro U, Campbell KP, et al. Alpha6beta4 integrin and dystroglycan cooperate to stabilize the myelin sheath. J Neurosci. 2008;28:6714-9.

74. Jones RG, Li X, Gray PD, Kuang J, Clayton F, Samowitz WS, Madison BB, Gumucio DL, Kuwada SK. Conditional deletion of beta1 integrins in the intestinal epithelium causes a loss of hedgehog expression, intestinal hyperplasia, and early postnatal lethality. J Cell Biol. 2006;175:505-14.

75. Albuquerque C, Breukel C, van der Luijt R, Fidalgo P, Lage P, Slors FJ, Leitao $C N$, Fodde R, Smits R. The 'just-right' signaling model: APC somatic mutations are selected based on a specific level of activation of the betacatenin signaling cascade. Hum Mol Genet. 2002;11:1549-60.

76. Lewis A, Segditsas S, Deheragoda M, Pollard P, Jeffery R, Nye E, Lockstone $H$, Davis H, Clark S, Stamp G, et al. Severe polyposis in Apc(1322T) mice is associated with submaximal Wnt signalling and increased expression of the stem cell marker Lgr5. Gut. 2010;59:1680-6.

77. Hu Y, Yan C, Mu L, Huang K, Li X, Tao D, Wu Y, Qin J. Fibroblast-derived Exosomes contribute to Chemoresistance through priming Cancer stem cells in colorectal Cancer. PLoS One. 2015;10:e0125625.

78. Brabletz T, Jung A, Spaderna S, Hlubek F, Kirchner T. Opinion: migrating cancer stem cells - an integrated concept of malignant tumour progression. Nat Rev Cancer. 2005;5:744-9.

79. Yoshimura K, Meckel KF, Laird LS, Chia CY, Park JJ, Olino KL, Tsunedomi R, Harada T, lizuka N, Hazama S, et al. Integrin alpha2 mediates selective metastasis to the liver. Cancer Res. 2009;69:7320-8.

\section{Publisher's Note}

Springer Nature remains neutral with regard to jurisdictional claims in published maps and institutional affiliations.

\section{Ready to submit your research? Choose BMC and benefit from:}

- fast, convenient online submission

- thorough peer review by experienced researchers in your field

- rapid publication on acceptance

- support for research data, including large and complex data types

- gold Open Access which fosters wider collaboration and increased citations

- maximum visibility for your research: over $100 \mathrm{M}$ website views per year

At BMC, research is always in progress.

Learn more biomedcentral.com/submissions 